\title{
AP2/ERF transcription factor NbERF-IX-33 is involved in the regulation of phytoalexin production for the resistance of Nicotiana benthamiana to Phytophthora infestans
}

1 Sayaka Imano ${ }^{1}$, Mayuka Fushimi ${ }^{1}$, Maurizio Camagna ${ }^{1}$, Akiko Tsuyama-Koike ${ }^{1}$, Hitoshi 2 Mori $^{1}$, Akira Ashida ${ }^{1}$, Aiko Tanaka ${ }^{1}$, Ikuo Sato ${ }^{1}$, Sotaro Chiba ${ }^{1}$, Kazuhito Kawakita ${ }^{1}$, Makoto

3 Ojika ${ }^{1}$, Daigo Takemoto ${ }^{1^{*}}$

$4{ }^{1}$ Graduate School of Bioagricultural Sciences, Nagoya University, Chikusa, Nagoya, 464-8601, 5 Japan

* Correspondence:

Daigo Takemoto

dtakemo@agr.nagoya-u.ac.jp

Keywords: Capsidiol, ERF, Ethylene, Nicotiana benthamiana, Phytoalexin, Phytophthora infestans.

\begin{abstract}
Plants recognize molecular patterns unique to a certain group of microbes to induce effective resistance mechanisms. Elicitins are secretory proteins produced by plant pathogenic oomycete genera including Phytophthora and Pythium. Treatment of INF1 (an elicitin produced by $P$. infestans) induces a series of defense responses in Nicotiana species, including reactive oxygen species (ROS) production, transient induction of ethylene production, hypersensitive cell death and accumulation of the sesquiterpenoid phytoalexin capsidiol. In this study, we analyzed the expression profiles of $N$. benthamiana genes after INF1 treatment by RNAseq analysis. Based on their expression patterns, $N$. benthamiana genes were categorized into 20 clusters and 4,761 (8.3\%) out of 57,140 genes were assigned to the clusters for INF1-induced genes. All genes encoding enzymes dedicated to capsidiol production, 5-epi-aristolochene (EA) synthase ( $N b E A S, 10$ copies) and EA dehydrogenase (NbEAH, 6 copies), and some genes for ethylene production, such as 1aminocyclopropane 1-carboxylate (ACC) synthase $(\mathrm{NbACS})$ and ACC oxidase $(\mathrm{NbACO})$, were significantly upregulated by INF1 treatment. Analysis of NbEAS1 and NbEAS4 promoters revealed that AGACGCC (GCC box-like motif) is the essential cis-element required for INF1-induced expression of $N b E A S$ genes. Given that the GCC box is known to be targeted by ERF (ethyleneresponsive factor) transcription factors, we created a complete list of $N$. benthamiana genes encoding AP2/ERF family transcription factors, and identified 45 out of 337 AP2/ERF genes in the clusters for INF1-induced genes. Among INF1-induced $N b E R F$ genes, silencing of $N b E R F-I X-33$ compromised resistance against $P$. infestans and INF1-induced production of capsidiol. Recombinant NbERF-IX33 protein can bind to the promoter sequence of NbEAS4, suggesting that NbERF-IX-33 is a transcription factor directly regulating the expression of genes for phytoalexin production.
\end{abstract}




\section{INTRODUCTION}

Plants recognize a variety of molecules derived from pathogens, including components of microbial cell walls, membranes and secreted proteins (Ranf, 2017; Monjil et al., 2021). Elicitins are small secretory proteins produced by plant pathogenic oomycete genera such as Phytophthora, Pythium and Hyaloperonospora (Tyler, 2002; Takemoto et al., 2005; Cabral et al., 2011). Treatment with elicitins induces typical defense responses such as hypersensitive cell death, expression of $P R$ genes and production of phytoalexins (Milat et al., 1991; Bonnet et al., 1996; Matsukawa et al., 2013). Reports of plant species and cultivars distinctly responsive to elicitins include Nicotiana spp., some cultivars of Brassica rapa and Raphanus sativus, and Solanum microdontum (Bonnet et al., 1996; Takemoto et al., 2005; Du et al., 2015). Elicitins from different Phytophthora species elicit defense responses in these plants responsive to elicitin, indicating that responsive plants recognize elicitins as a molecular pattern of oomycete pathogens. The most virulent isolates of Phytophthora parasitica (syn. P. nicotianae) have lost the production of canonical elicitins, or downregulate the expression of the elicitin gene during infection (Ricci et al., 1992; Colas et al., 2001), indicating that the downregulation of elicitin represents a strategy for the pathogen to avoid recognition by the host plant. Elicitins are considered as an essential factor for the reproduction of Phytophthora and Pythium species (Blein et al., 2002). Elicitins contain a hydrophobic pocket similar to a lipid transfer protein and can bind to phytosterols to take up sterols from plant plasma membranes (Boissy et al., 1996; Mikes et al., 1998). Procuring sterols from the plant plasma membrane may be the primary function of elicitins, which could explain why elicitins are essential for reproduction, as Phytophthora and Pythium species are unable to produce sterols themselves (Hendrix, 1970).

Potato late blight caused by Phytophthora infestans is one of the most devastating and economically important plant diseases. $P$. infestans was the causal agent of the Irish potato famine in the 1840s, and even in present times, worldwide yield losses caused by this pathogen exceed $\$ 6$ billion per year (Haverkort et al., 2008). P. infestans has at least six genes for elicitins (Jiang et al., 2006), and INF1 is the most abundantly produced elicitin. Although INF1 treatment does not elicit a noticeable defense response in most Solanum species, including potato (S. tuberosum), a receptorlike protein ELR was isolated from the wild potato $S$. microdontum, which is able to recognize INF1 and induce a defense response (Du et al., 2015). Recognition of INF1 is important for the non-host resistance of Nicotiana benthamiana to $P$. infestans. Gene silencing of infl in $P$. infestans enhanced the virulence of the pathogen on $N$. benthamiana (Kamoun et al., 1998), suggesting that recognition of INF1 is essential for the defense induction in $N$. benthamiana against $P$. infestans. However, in contrast to S. microdontum, the gene for the recognition of INF1 has not yet been identified in $N$. benthamiana.

$N$. benthamiana is an ideal model host plant to study the molecular mechanisms underlying nonhost resistance of Solanaceae plants against $P$. infestans. Previously, we performed virus-induced gene silencing (VIGS)-based screenings to isolate genes essential for the resistance of $N$. benthamiana against $P$. infestans (Matsukawa et al., 2013; Ohtsu et al., 2014; Shibata et al., 2016; Takemoto et al., 2018; Mizuno et al., 2019a). So far, thirty-three genes have been identified from approx. 3,000 randomly gene-silenced plants as the essential genes for full resistance of $N$. benthamiana against $P$. infestans. Besides random gene silencing, $N$. benthamiana can also readily be used for targeted gene-silencing to further examine the involvement of homologs of known defense-related genes in the resistance to P. infestans (Shibata et al., 2010, 2011, 2016).

A major group of genes isolated from the VIGS screening are genes coding for enzymes in the mevalonate (MVA) pathway, for the production of sterols and a wide variety of isoprenoid-derived secondary metabolites (Shibata et al., 2016). In addition, silencing of genes for NbEAS (5-epiaristolochene synthase) and $\mathrm{NbEAH}$ (5-epi-aristolochene dihydroxylase), encoding the enzymes dedicated to the production of the sesquiterpenoid phytoalexin capsidiol, compromised resistance of 
$N$. benthamiana to $P$. infestans. Six genes related to ethylene biosynthesis were also isolated as essential genes for the resistance of $N$. benthamiana against $P$. infestans. Expression of $N b E A S$ and $N b E A H$ genes was induced by the treatment with ethylene, and suppressed by gene silencing of NbEIN2, a central regulator of ethylene signaling, indicating that phytoalexin production is regulated by ethylene in $N$. benthamiana (Shibata et al., 2010, 2016; Ohtsu et al., 2014). However, the detailed mechanism of how ethylene signaling regulates the genes for phytoalexin production in $N$. benthamiana has not been fully elucidated.

In a previous study, we performed RNAseq analysis of $N$. benthamiana treated with INF1 at a single time point ( $24 \mathrm{~h}$ after treatment, Rin et al., 2020). While these findings were insightful, they merely represent a single snapshot and are therefore insufficient to capture the dynamic expression processes during pathogen defense. In the present study, we performed RNAseq analysis of INF1 elicited $N$. benthamiana for various time points to obtain more detailed expression profiles of the genes involved in defense response. The activity of $N b E A S$ gene promoters was analyzed to identify the cis-acting element essential for the INF1-induced expression of $N b E A S$ genes. Moreover, we created a complete catalog of the AP2/ERF (APETALA 2/ethylene-responsive element binding factor) transcription factor genes for $N$. benthamiana and analyzed their expression profiles to identify the transcription factor directly regulating the expression of $N b E A S$ genes.

\section{MATERIALS AND METHODS}

\section{Biological Materials, Growth Conditions, Inoculation and Treatment}

$N$. benthamiana line SNPB-A5 (Shibata et al., 2016) was grown in a growth room at $23^{\circ} \mathrm{C}$ with 16 $\mathrm{h}$ of light per day. $P$. infestans isolate 08YD1 (Shibata et al., 2010) was maintained on rye-media at $20^{\circ} \mathrm{C}$ in the dark. Preparation of zoospore suspension of $P$. infestans and inoculation of $N$. benthamiana leaves (approx. 45 days old) with a zoospore suspension of $P$. infestans was performed as described previously (Shibata et al., 2010). INF1 elicitor was prepared from Escherichia coli (strain DH5 $\alpha$ ) carrying an expression vector for INF1, pFB53, as previously reported (Kamoun et al., 1997; Shibata et al., 2010). N. benthamiana leaves were treated with $150 \mathrm{nM}$ INF1 solution as previously described (Shibata et al., 2010).

\section{Measurement of Reactive Oxygen Species (ROS) Production.}

The relative intensity of ROS generation was determined by counting photons from L-012mediated chemiluminescence. For the detection of ROS production at early time points (for Figure 1A), $N$. benthamiana leaf discs (4 $\mathrm{mm}$ in diameter) were excised with a cork borer and floated on 100 $\mu \mathrm{l}$ distilled water in a 96-well microplate (Nunc 96F microwell white polystyrene plate, Thermo Fisher Scientific, Waltham, MA, USA) overnight in a growth chamber $\left(23^{\circ} \mathrm{C}\right)$. Just before the measurement, water in each well was replaced with $50 \mu 1$ of water or $150 \mathrm{nM} \mathrm{INF} 1$ containing $1 \mathrm{mM}$ L-012 (Wako Pure Chemical, Osaka, Japan). Chemiluminescence was measured by a multiplate reader (TriStar LB941; Berthold Technologies, Bad Wildbad, Germany). For the detection of ROS production at relatively later time points (for Figure 1B), N. benthamiana leaves were infiltrated with water or $150 \mathrm{nM} \mathrm{INF1} \mathrm{and} \mathrm{incubated} \mathrm{in} \mathrm{a} \mathrm{growth} \mathrm{room} \mathrm{at} 23^{\circ} \mathrm{C}$, and $0.5 \mathrm{mM} \mathrm{L}-012$ was allowed to infiltrate to the intercellular space of leaves before the measurement. ROS production was measured as chemiluminescence using Lumino Graph II EM (ATTO, Tokyo, Japan).

\section{Ion Leakage Assay}

The severity of cell death induced by INF1 treatment was quantified by Ion leakage assay as previously described (Mizuno et al., 2019b). Four leaf disks (5 mm diameter) of $N$. benthamiana 
treated with water or $150 \mathrm{nM}$ INF1 were floated on $1 \mathrm{ml}$ water under light condition for $6 \mathrm{~h}$. Ion conductivity was measured by LAQUAtwin compact conductivity meter (Horiba, Kyoto, Japan).

\section{Quantitative Analysis of Phytoalexin Production by Liquid Chromatography (LC)}

For the quantification of phytoalexins shown in Figure $1 \mathrm{C}$, leaves of $N$. benthamiana (50 mg, fresh weight) treated with elicitor were soaked in $1.5 \mathrm{ml}$ ethyl acetate with gentle shaking for an hour. After the evaporation of ethyl acetate, the dried substances were redissolved in $100 \mu 1$ of $1: 1$ acetonitrile: water (v/v) and produced phytoalexins were measured by LC/MS (Accurate-Mass QTOF LC/MS 6520, Agilent Technologies, Santa Clara, CA, USA) with ODS column Cadenza CDC18, $75 \times 2 \mathrm{~mm}$ (Imtakt, Kyoto, Japan). For the quantification of phytoalexins shown in Figure 8C, extraction and quantification of capsidiol were performed as previously described (Matsukawa et al., 2013).

\section{RNA-seq Analysis and Clustering of $N$. benthamiana Genes}

Total RNA was extracted from $N$. benthamiana leaves using the RNeasy Plant Mini Kit (QIAGEN, Hilden, Germany). Libraries were constructed using KAPA mRNA Capture Kit (Roche Diagnostics, Tokyo, Japan) and MGIEasy RNA Directional Library Prep Set (MGI, Shenzhen, China), and sequenced on DNBSEQ-G400RS (MGI) with 150 bp paired-end protocol. The RNA-seq reads were filtered using trim-galore v.0.6.6 (Martin, 2011, bioinformatics.babraham.ac.uk) and mapped to the $N$. benthamiana genome (ver. 1.0.1, https://solgenomics.net, Bombarely et al., 2012) using HISAT2 v.2.2.1 (Kim et al., 2019) and assembled via StringTie v.2.1.7 (Kovaka et al., 2019). Significant differential expression was determined using DESeq2 v.1.32.0 (Love et al., 2014). All software used during RNA-seq analysis was run with default settings. For the clustering of $N$. benthamiana genes, K-means clustering was performed using TimeSeriesKMeans from the tslearn (v.0.5.2) Python package $(\mathrm{k}=20)$. Before clustering, the log2-fold expressions for each gene were preprocessed, so that the mean expression of each time series equaled 0. RNA-seq data reported in this work are available in GenBank under the accession number DRA013037.

167

\section{Quantitative Analysis of Ethylene Production by Gas Chromatography (GC)}

Ethylene production was quantified as previously described (Matsukawa et al., 2013). Leaf disks (10 $\mathrm{mm}$ in diameter) of $N$. benthamiana were excised with a cork borer and placed in a 5-ml GC vial sealed with a rubber plunger. After $3 \mathrm{~h}$ of incubation, a 1-ml air sample in the vial was collected using a glass syringe. The quantity of ethylene in the collected air was measured using a gas chromatograph equipped with a flame thermionic detector (GC-353, GL Sciences, Tokyo, Japan) and CP-Permabond Q column (Varian Inc., Middelburg, Netherlands). GC was performed with column temperature at $50^{\circ} \mathrm{C}$, injection temperature at $120^{\circ} \mathrm{C}$, and detector temperature at $150^{\circ} \mathrm{C}$ using $\mathrm{N}_{2}$ as the carrier gas.

\section{Construction of Vectors and Transformation of Agrobacterium tumefaciens}

Base vectors, primer sets and templates for the PCR amplification of DNA fragments for vector construction are listed in Supplementary Table 1. Sequences of primers used for the construction of vectors are listed in Supplementary Table 2. Gene fragments were amplified using PrimeStar HS DNA polymerase (Takara Bio, Kusatsu, Japan) and cloned into the vector using In-Fusion HD Cloning Kit (Takara Bio). Gene fragments in pTV00 vectors for VIGS induction were assessed using the SGN VIGS tool (Fernandez-Pozo et al., 2015) to exclude unexpected off-target effects. Vectors for transient expression or VIGS were transformed into A. tumefaciens (strain C58C1) by electroporation with a MicroPulser electroporator (Bio-Rad, Hercules, CA, USA). A. tumefaciens transformants were selected on LB media containing $50 \mu \mathrm{g} / \mathrm{ml}$ rifampicin, $50 \mu \mathrm{g} / \mathrm{ml}$ kanamycin, and

$18850 \mu \mathrm{g} / \mathrm{ml}$ ampicillin at $28^{\circ} \mathrm{C}$. 


\section{Transient Gene Expression by Agroinfiltration}

A. tumefaciens carrying expression vectors (pNPP40 and pNPP243 derivatives, Supplementary Table S1) were cultured to saturation in $\mathrm{LB}$ medium at $28^{\circ} \mathrm{C}$ and bacterial cells were collected by centrifugation at $16,000 \mathrm{x}$ g for $1 \mathrm{~min}$. The bacterial cells were then resuspended in MMA infiltration solution to a final $\mathrm{OD}_{600}$ of 0.5 (MMA: $5 \mathrm{mg} / \mathrm{ml} \mathrm{MS} \mathrm{salts,} 1.95 \mathrm{mg} / \mathrm{ml} \mathrm{MES,} 20 \mathrm{mg} / \mathrm{ml}$ sucrose, 200 $\mu \mathrm{M}$ acetosyringone, $\mathrm{pH}=5.6$ ) and incubated at $28^{\circ} \mathrm{C}$ for $2 \mathrm{~h}$. The suspensions were allowed to infiltrate the intercellular space of $N$. benthamiana leaves using a syringe without a needle.

\section{Quantitative RT-PCR}

Total RNAs were isolated from $N$. benthamiana leaves using TRIzol Reagent (Thermo Fisher Scientific) and cDNA synthesis was conducted using ReverTra Ace- $\alpha$ - (Toyobo, Osaka, Japan). Quantitative RT-PCR (qRT-PCR) analysis was performed using LightCycler Quick System 350S (Roche Applied Science, Penzberg, Germany) with Thunderbird SYBR qPCR Mix (Toyobo). The expression of $N$. benthamiana $E F-1 \alpha$ gene was used as an internal standard. Gene-specific primers used for expression analysis were listed in Supplementary Table S2.

\section{Fluorescence Microscopy}

To visualize the activation of NbEAS4 promoter by GFP marker, fluorescence images were recorded using BX51 fluorescence microscope (Olympus, Tokyo, Japan) equipped with color CMOS camera Wraycam-NF500 (Wraymer, Osaka, Japan).

\section{Virus-induced Gene Silencing (VIGS)}

The induction of VIGS was carried out as previously reported (Ratcliff et al., 2001; Shibata et al., 2010). A. tumefaciens strain GV3101 delivering the binary TRV RNA1 construct pBINTRA6, and the TRV RNA2 vector pTV00 or its derivatives (Supplementary Table 1), were cultured to saturation in LB media. Bacterial cells were collected by centrifugation at $16,000 \times \mathrm{g}$ for $1 \mathrm{~min}$. The bacterial cells were then resuspended in $10 \mathrm{mM}$ MES-NaOH ( $\mathrm{pH}$ 5.6), $10 \mathrm{mM} \mathrm{MgCl} \mathrm{M}_{2}$ and $150 \mu \mathrm{m}$ acetosyringone (final $\mathrm{OD}_{600}=0.5$ ) and incubated at room temperature for $2 \mathrm{~h}$. The cultures were mixed in a 1:1 ratio (RNA1/RNA2), to infiltrate leaves of $N$. benthamiana using a syringe without a needle. After 3-4 weeks of infiltration, the upper leaves of the inoculated plants were used for experiments.

\section{Expression of Recombinant Proteins in E. coli}

The MBP-NbERF-IX-33a fusion protein was prepared using the pMAL Protein Fusion and Purification System (New England Biolabs). E. coli cells (DH5) carrying pMAL-c5x (New England Biolabs) or pMAL-NbERF-IX-33a were cultured overnight at $37^{\circ} \mathrm{C}$ in rich medium ( $1 \%$ tryptone, $0.5 \%$ yeast extract, $0.5 \% \mathrm{NaCl}, 0.2 \%$ glucose) containing $100 \mu \mathrm{g} / \mathrm{ml}$ ampicillin and grown until the optical density $\left(\mathrm{OD}_{600}=0.6\right)$. Production of the protein was induced by adding $0.3 \mathrm{mM}$ IPTG for $5 \mathrm{~h}$. The culture $(20 \mathrm{ml})$ was centrifuged to collect $E$. coli cells. The cells were then resuspended in $6 \mathrm{ml}$

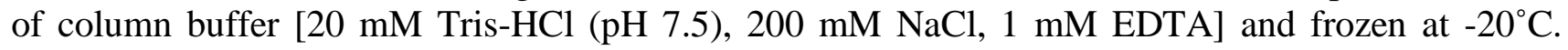
Frozen cells were thawed on ice and then homogenized using a sonicator (Sonicator cell disruptor Model W-225R, Heat Systems-Ultrasonics Inc., Plainview, NY, USA). The crude cell extracts were centrifuged at $10,000 \mathrm{x} \mathrm{g}, 4^{\circ} \mathrm{C}$ for $5 \mathrm{~min}$, and the supernatant was adsorbed to a $0.4 \mathrm{ml}$ amylose resin (New England Biolabs) at $4^{\circ} \mathrm{C}$. The column was washed with $4 \mathrm{ml}$ of column buffer, followed by elution of the fusion protein with $1 \mathrm{ml}$ of column buffer containing $10 \mathrm{mM}$ maltose. The fractions of purified proteins were assessed by SDS-PAGE and eluted fractions were dialyzed against water with the dialysis tubing (SnakeSkin dialysis tubing, $3.5 \mathrm{kD}$ molecular mass cutoff, Thermo Fisher Scientific) overnight at $4^{\circ} \mathrm{C}$. 
238

239

240

241

242

243

244

245

246

247

248

249

250

251

252

253

254

255

256

257

258

259

260

261

262

263

264

265

266

267

268

269

270

271

272

273

274

275

276

277

278

279

280

281

282

283

284

285

286

\section{Electrophoresis Mobility Shift Assay (EMSA)}

Electrophoresis mobility shift assay Kit (Thermo Fisher Scientific) was used to detect binding of NbERF-IX-33a to the NbEAS4 promoter. The DNA fragment of NbEAS4 promoter (30 ng) and MBP or MBP- NbERF-IX-33a proteins $(1 \mu \mathrm{g})$ were mixed and incubated in $10 \mu \mathrm{l}$ binding buffer [125 $\mathrm{mM}$ HEPES-KOH, $20 \mathrm{mM} \mathrm{KCl,} 0.5 \mathrm{mM}$ EDTA, 50\% (v/v) glycerol, $5 \mathrm{mM} \mathrm{DTT,} \mathrm{pH7.5]} \mathrm{for} 20 \mathrm{~min}$ at $4^{\circ} \mathrm{C}$. $2 \mu 1$ of $6 \mathrm{x}$ loading buffer was added to stop the reaction. The complexes were resolved on $6 \%$ non-denaturing polyacrylamide gels (Thermo Fisher Scientific) and stained with SYBR green dye.

\section{RESULTS}

\section{Induction of ROS Production, Phytoalexin Accumulation and Cell Death in N. benthamiana treated with INF1}

Prior to the RNAseq analysis of $N$. benthamiana genes induced by INF1 treatment, typical defense responses induced by INF1 treatment were observed under our experimental conditions. Induction of reactive oxygen species (ROS) production is one of the common phenomena observed during the induction of plant disease resistance. Using small leaf disks of $N$. benthamiana, transient ROS production was detected within $60 \mathrm{~min}$ after INF1 treatment (Figure 1A). Significant increase in ROS production was also observed at $12 \mathrm{~h}$ and $24 \mathrm{~h}$ after treatment (Figure 1B). These results are consistent with previous studies that reported biphasic ROS production in plants during the plant defense (Chai and Doke, 1987; Levine et al., 1994; Yuan et al., 2021). The production of sesquiterpenoid phytoalexins, capsidiol, debneyol and capsidiol 3-acetate were significantly increased at $24 \mathrm{~h}$ and $48 \mathrm{~h}$ after treatment with $150 \mathrm{nM} \mathrm{INF1}$, while the production of phytoalexins was below the detection limit at earlier times (Figure 1C). Induction of visible cell death became obvious at around $48 \mathrm{~h}$ after INF1 treatment, while the increase of ion leakage from the INF1-treated plant tissue (indicating the initiation of cell death) was already detectable at $24 \mathrm{~h}$ (Figure 1D).

\section{Clustering of $N$. benthamiana Genes Based on Their Expression Patterns after INF1 Treatment}

To investigate the expression profile of all $N$. benthamiana genes during the induction of defense responses by INF1 treatment, RNA-seq analysis was performed for $N$. benthamiana leaves treated with water or INF1 at $0,3,6,12$ and $24 \mathrm{~h}$ after the treatment. Based on their time dependent expression patterns, all genes were categorized into 20 groups using K-means clustering. Among the $57,140 \mathrm{~N}$. benthamiana annotated genes, 14,390 genes (25.2\%) were not assigned to any cluster because no expression could be determined at any point in time. Among the 20 clusters, clusters 2, 4, 10, 14 and 16 were the clusters that contained significantly more INF1-inducible genes, and these include 4,761 (8.3\%) out of 57,140 genes (Figure 2). Especially, the genes in cluster 14 (contains 336 genes) were significantly up-regulated after INF1 treatment, with more than 300-fold induction at 24 $\mathrm{h}$ (average in cluster 14) compared to control (Figure 2). The lists of genes in clusters for INF1induced N. benthamiana genes are shown in Supplementary Tables 3-7.

Cluster 14 includes all 10 copies of $\mathrm{NbEAS}$ and 5 out of $6 \mathrm{NbEAH}$ genes which are genes specific for the capsidiol production (Figure 3, Supplementary Table 8). Only negligible expression of genes in cluster 14 was observed in control and water-treated leaves, indicating that genes specifically involved in the production of phytoalexin are under strict regulation.

In contrast, most genes for the enzymes in the MVA pathway (required for the production of capsidiol precursor, farnesylpyrophosphate, FPP) were categorized into other clusters as most of them have a basal constitutive expression in control and water treated leaves, probably reflecting that FPP is a common precursor of isoprenoids and phytosterol (Supplementary Table 9, Figure 4). Among five acetyl-CoA thiolase (ACAT) genes, only the expression of NbACATIb was highly 
287

288

289

290

291

292

293

294

295

296

297

298

299

300

301

302

303

304

305

306

307

308

309

310

311

312

313

314

315

316

317

318

319

320

321

322

323

324

325

326

327

328

329

330

331

332

333

334

335 upregulated from an early time point. Out of five 3-hydroxy-3-methylglutaryl-CoA (HMG-CoA) synthase genes, NbHMGSIa and $1 b$ were found to be induced by INF1 treatment. Similarly, among five $H M G-C o A$ reductase ( $N b H M G R$ ) genes, $N b H M G R 2$ and $1 a$ were significantly upregulated by INF1 treatment. In the case of genes for mevalonate-5-kinase ( $N b M V K, 2$ copies), phosphomevalonate kinase (NbPMVK, 2 copies), and mevalonate-5-pyrophosphate decarboxylase (NbMVD, 2 copies), they were all upregulated by INF1 treatment. Likewise, two out of four genes encoding isopentenyl pyrophosphate (IPP) isomerase, $N b I P P I 1 b$ and $2 a$, were appeared to mainly contribute to the activation of the conversion of IPP to dimethylallyl pyrophosphate (DMAPP) during the induction of capsidiol production, while stable and constant expression was observed for $N b I P P I 2 b$. Finally, among 4 genes for FPP synthase (NbFPPS), NbFPPS1a, NbFPPS1b, NbFPPS $2 b$ were significantly upregulated (Figure 4).

\section{Identification of Cis-element for INF1-induced Expression of $\mathrm{NbEAS}$ Genes}

To further investigate the strict regulation of $N b E A S$ genes, we analyzed promoters of $N b E A S 1$ and NbEAS4 in this study. To evaluate the promoter activity of these genes, plasmid vectors containing the GFP gene under the control of NbEAS1 or NbEAS4 promoter ( $\mathrm{P}_{-} N b E A S 1: G F P$ and P_NbEAS4:GFP) were transiently transformed into $N$. benthamiana leaves by Agroinfiltration. Basal expression of GFP was detected after agroinfiltration using A. tumefaciens containing these promoter:GFP vectors under fluorescence microscopy, presumably because plant defense was weakly induced by the infection with $A$. tumefaciens. Nonetheless, the relative expression levels of GFP were significantly enhanced by treatment with INF1. When the promoter length exceeded $260 \mathrm{bp}$ upstream from the start codon (-260) was used, GFP expression was induced by INF1 treatment for both NbEAS1 and NbEAS4 promoters. When the NbEAS1 promoter was shortened to a length of $230 \mathrm{bp}$, INF1-induced GFP expression was no longer observed, whereas the NbEAS4 promoter remained functional. INF1-induced GFP expression ceased however when the NbEAS4 promoter was further trimmed to a length of 200 bp (Figure 5A). From these findings it follows that the regions essential for the induction of NbEAS1 and NbEAS4 expression by INF1 are located between -260 and -230 of the $N b E A S 1$ promoter, and between -230 and -200 of the NbEAS4 promoter. In the corresponding region of $N b E A S 1$ and $N b E A S 4$ promoter, a sequence similar to the ethylene-responsive element GCC box (AGCCGCC), AGACGCC, was identified (Figure 5B). To confirm the importance of the GCC box-like motif on the promoter activity, we introduced mutations to the motif in the P_NbEAS4:GFP vector, which led to substitutions of $\mathrm{G}$ to T (ATACTCC). We designated the vector carrying the mutated GCC box-like motif as $\mathrm{P} \_N b E A S 4$ (TT). G to T mutations in the GCC box-like motif significantly reduced both the fluorescence of GFP in $N$. benthamiana leaves $48 \mathrm{~h}$ after Agroinfiltration, as well as the relative expression level of GFP (Figure 5C), indicating that the GCC-box like motif is the cis-acting element essential for the INF1-induced expression of EAS4. GCC-box like motifs can be found in most of $N b E A S$ and $N b E A H$ genes (Supplementary Figure 1), while the GCC-box was not found in the promoter regions of genes in the MVA pathway, indicating that production of the capsidiol precursor FPP and capsidiol production are probably controlled via distinctive regulatory mechanisms.

\section{Expression Profile of $\boldsymbol{N}$. benthamiana Genes for Enzymes Involved in the Ethylene Production}

Previously, we have isolated three genes essential for resistance of $N$. benthamiana to $P$. infestans belonging to the methionine cycle, which is related to the production of the ethylene precursor SAM (S-adenosyl-L-methionine) (Shibata et al., 2016, Figure 6). In this study, we created a list of genes for putative enzymes in the methionine cycle and for ethylene biosynthesis (Supplementary Table 10) to investigate their expression profiles after INF1 treatment. Given that $N$. benthamiana has an allopolyploid genome (Goodin et al., 2008), two highly homologous genes were frequently found, and which were predicted to be derived from the two ancestral Nicotiana species of N. benthamiana. 
336

337

338

339

340

341

342

343

344

345

346

347

348

349

350

351

352

353

354

355

356

357

358

359

360

361

362

363

364

365

366

367

368

369

370

371

372

373

374

375

376

377

378

379

380

381

382

383

384

Such highly homologous pairs of genes were named by appending either $a$ or $b$ to the gene name (Matsukawa et al., 2013; Shibata et al., 2016; Rin et al., 2020). For example, the highly homologous genes for cystathionine $\gamma$-synthase (CGS) were designated as NbCGSIa and NbCGSIb.

Among the 32 putative methionine cycle genes (Supplementary Table 10), only two genes (NbCGSla and NbSAM4a) were moderately upregulated by INF1 treatment (Figure 6). For genes encoding enzymes specific for ethylene production, 24 1-aminocyclopropane-1-carboxylate (ACC) synthase $(N b A C S)$ and 16 ACC oxidase $(N b A C O)$ genes were identified in the genome of $N$. benthamiana (Supplementary Table 10). Among the 24 NbACS genes, three genes, NbACS1, $2 a$ and 4 were upregulated by INF1 treatment. Four out of the $16 \mathrm{NbACO}$ genes were categorized as INF1 inducible genes, of which NbACOla, $1 b$ and 4 were highly upregulated (Figure 6). Ethylene production was induced within $3 \mathrm{~h}$ after treatment of INF1, and gene silencing of $N b A C O$ genes (using a conserved region of $N b A C O$ genes) compromised INF1 induced production of ethylene (Supplementary Figure 2A). NbACO-silenced $N$. benthamiana, as well as NbEIN2-silenced plants, showed enhanced disease symptoms by $P$. infestans compared with the control plants (Supplementary Figure 2B), confirming the importance of ethylene production in disease resistance of $N$. benthamiana.

\section{$N$. benthamiana NbERF-IX-33 is Involved in the Production of Capsidiol and Resistance to $P$. infestans}

The ERF (ethylene response factor) family transcription factors are known to be involved in the responses of plants to a number of environmental stresses (Müller and Munné-Bosch, 2015). Previous studies indicated that some ERF transcription factors can directly bind to a cis-acting element, typically the GCC box (AGCCGCC), to respond to pathogen attack (Ohme-Tagaki and Shinshi, 1995), while some studies also indicated that another member of the ERF transcription factors can bind to the dehydration responsive element (DRE, typically TACCGAC) to respond to drought stress (Yamaguchi-Shinozaki and Shinozaki, 1994). To investigate the activation of ethylene signaling during the defense induction in $N$. benthamiana, we created a complete list of predicted genes for AP2/ERF transcription factors found in the genome of $N$. benthamiana, using $147 \mathrm{~A}$. thaliana AP2/ERF (Nakano et al., 2006) as queries for Blastp search against $N$. benthamiana predicted proteins (Sol genomics, Fernandez-Pozo et al., 2015). In the genome of $N$. benthamiana, we found 337 genes predicted to encode AP2/ERF transcription factors (Supplementary Tables 11 and 12). Based on the phylogenetic analysis of A. thaliana and $N$. benthamiana AP2/ERF genes, 47 genes were assigned to the AP2 family (including Soloist I) and 7 genes were classified in the RAV family (Figure 7 and Supplementary Table 11). The $283 N$. benthamiana ERF genes were classified into 10 major groups (ERF-I to -X as in the case of A. thaliana, Nakano et al., 2006) and two small subgroups (Soloist II and III). Almost all groups of AP2/ERF transcription factors have corresponding members in Arabidopsis and N. benthamiana, suggesting that their functions are conserved in these two dicotyledonous plants. Among these, group ERF-IX contained the highest number of elicitor-responsive genes, and 22 out of the 34 genes (assigned to a cluster) were classified into INF1-induced clusters (Figure 7 and Supplementary Table 12). Because a substantial number of genes for NbERF were elicitor-inducible, it was expected that these NbERFs may have redundant functions in the induction of disease resistance.

To investigate the role of ERF-type transcription factors in the production of capsidiol during the induction of disease resistance, two $N b E R F$ were selected for further analysis in this study. NbERF$I X-33 a$ (Niben101Scf00454g04003, cluster 4) whose expression level (FPKM value) was the highest among all AP2/ERF genes at $12 \mathrm{~h}$ after INF1 treatment, and NbERF-IX-16a (Niben101Scf01212g03005, cluster 14) whose expression level was the highest at $24 \mathrm{~h}$ after INF1 treatment (Figure 8A). For the specific silencing of these target genes, silencing vectors based on unique sequences of NbERF-IX-33 and NbERF-IX-16 were constructed. Both NbERF-IX-33 and 
385

386

387

388

389

390

391

392

393

394

395

396

397

398

399

400

401

402

403

404

405

406

407

408

409

410

411

412

413

414

415

416

417

418

419

420

421

422

423

424

425

426

427

428

429

430

431

432

433
NbERF-IX-16 occur in the form of highly similar $a$ and $b$ homologs, as described above. Due to the high sequence similarity of $a$ and $b$ homologs, a functional redundancy is to be expected and we therefore designed the silencing vectors to target the $a$ and $b$ homologs of both genes. Analysis using the SGN VIGS tool (Fernandez-Pozo et al., 2015) confirmed that the constructed vectors are specifically targeting $N b E R F-I X-33$ and $N b E R F-I X-16$ genes, and no potential off-target effect was detected.

NbERF-IX-33 and NbERF-IX-16-silenced N. benthamiana plants were inoculated with $P$. infestans and disease symptoms on control and gene-silenced plants were scored as visible development of disease symptoms. Within the first 5 days after the inoculation of $P$. infestans, there was no obvious development of disease symptoms for control and NbERF-IX-16-silenced plants was detected, whereas NbEIN2 - and NbERF-IX-33-silenced plants showed severe disease symptoms on the inoculated leaves (Figure 8B). To investigate the effect of the $N b E R F$ gene silencing of these $N b E R F$ on phytoalexin production, capsidiol was quantified in gene-silenced $N$. benthamiana. At $24 \mathrm{~h}$ after treatment with $150 \mathrm{nM}$ INF1, capsidiol was extracted from control and gene-silenced $N$. benthamiana leaves, and the amount of capsidiol produced was quantified using HPLC. While capsidiol production in NbERF-IX-16-silenced $N$. benthamiana was not significantly different from that of the control (TRV) plant, capsidiol amounts in NbERF-IX-33- and NbEIN2-silenced plants were reduced (Figure $8 \mathrm{C}$ ). These results indicated that $N b E R F-I X-33$ is a transcription factor essential for disease resistance through the production of capsidiol. The function of $N b E R F-I X-33$ appears to be partially compensated by other NbERF transcription factors in NbERF-IX-33 silenced plants, given that both disease resistance and capsidiol production were more pronounced in NbEIN2silenced plants.

To investigate whether NbERF-IX-33 acts directly on the NbEAS promoter, an electrophoresis mobility shift assay (EMSA) was conducted. To this end, we expressed and purified a recombinant fusion protein of maltose-binding protein (MBP) and NbERF-IX33 (MBP-NbERF-IX-33a) from $E$. coli (Supplementary Figure 3). The mobility shift assay was performed using the purified MBPNbERF-IX-33a protein with NbEAS4 promoter fragments. Mobility shift was observed for the $230 \mathrm{bp}$ NbEAS4 promoter fragment incubated with the NbERF-IX-33a protein, in contrast to the control experiment using MBP (Figure 8D). These results suggested that NbERF-IX-33 directly binds to the promoter region of NbEAS4 to increase the production of phytoalexin production.

\section{DISCUSSIONS}

Salicylic acid, jasmonic acid, and ethylene are commonly known as second messengers that play important roles in plant disease responses, but which plant hormones are essential for effective resistance induction varies among plant-pathogen combinations (Glazebrook, 2005). In general, salicylic acid plays an important role in the activation of defense against biotrophic and hemibiotrophic pathogens, while jasmonic acid and ethylene are usually implicated in the defense against necrotrophic pathogens. Since similarities in gene expression patterns have been noted between jasmonic acid and ethylene treatments in Arabidopsis (Schenk et al., 2000), both plant hormones are often regarded as having similar functions in disease resistance.

In the case of the interaction between $N$. benthamiana and hemibiotrophic $P$. infestans, it is presumed that ethylene, but not jasmonic acid, is the crucial plant hormone essential for the resistance against the pathogen. Although RNAseq analysis in this study detected the INF1-induced expression of some genes related to jasmonic acid production (data not shown), silencing of $\mathrm{NbCOI1,}$ a component of the jasmonic acid receptor, had no effect on the resistance of $N$. benthamiana to $P$. infestans (Shibata et al., 2010). The VIGS-based screening for defense-related genes identified six ethylene-related genes, but no gene involved in the production of jasmonic acid was isolated (Shibata et al., 2016). Thus, the importance of ethylene in P. infestans resistance of $N$. benthamiana is evident, but it has not been clarified how ethylene is involved in the induction of resistance. 
434

435

436

437

438

439

440

441

442

443

444

445

446

447

448

449

450

451

452

453

454

455

456

457

458

459

460

461

462

463

464

465

466

467

468

469

470

471

472

473

474

475

476

477

478

479

480

481
In this study, we investigated the expression profiles of all $N$. benthamiana genes in leaves after treatment with INF1, a PAMP of oomycete pathogens. Among the genes induced by INF1, those in cluster 14 showed a particularly clear upregulation by INF1 treatment (Figure 2). The main gene group included in cluster 14 was PR (pathogenesis-related) protein genes, such as $P R-1, P R-2(\beta-1,3-$ glucanase), PR-3 (chitinase), PR-4 and PR-5 (Osmotin) (Supplementary Table 6). In the previous VIGS screening for $N$. benthamiana genes required for the resistance against $P$. infestans, however, none of the genes for PR proteins were isolated from 3,000 randomly gene-silenced plants, in contrast, several genes involved in the capsidiol synthase had been identified (Shibata et al., 2016). Targeted gene silencing of PRI did not show any detectable effect on the resistance of $N$. benthamiana against $P$. infestans (Shibata et al., 2010). These results indicate that not all genes whose expression is induced by INF1 elicitor treatment necessarily function in $P$. infestans resistance. Nevertheless, cluster 14 contains genes for $N b E A S$ and $N b E A H$, which are required for the resistance of $N$. benthamiana to $P$. infestans. The expression of $N b E A S$ and $N b E A H$ was markedly induced by INF1 treatment, however, the expression of these genes was not stimulated at all by water treatment. (Figure 3). Since there is a trade-off between the expression of genes for disease resistance and plant growth (Denancé et al., 2013), it is important to strictly control the expression of genes involved in plant defense. In fact, many genes for photosynthesis (such as $r b c S$ and Lhcb genes) showed a tendency to be down-regulated in INF1-treated leaves (data not shown).

Promoter analysis of NbEAS1 and NbEAS4 revealed that the deletion of the GCC box-like sequence compromises the INF-induced expression of $N b E A S$ genes (Figure 5), indicating the direct regulation of phytoalexin production by ERF. Previous reports described the presence of a GCC box in the promoter region of the pepper and tobacco EAS genes (Maldonado-Bonilla et al., 2008), but its role in regulating EAS expression had not been analyzed. This study proves that the GCC box-like motif is indispensable for the induction of the $N b E A S$ gene, but does not rule out the possibility that other cis sequences are included in the promoter region. W-box $(\mathrm{C} / \mathrm{T}) \mathrm{TGCA}(\mathrm{C} / \mathrm{T})$ motifs, previously shown to correlate with the defense induction in $N$. benthamiana (Ishihama et al., 2011), are found in the promoter region of some $N b E A S$ and $N b E A H$ genes (Supplementary Figure 1). We also noticed that when we shortened the length of the NbEAS1 promoter from 400 to 300 (300 to 260 in NbEAS4) for the analysis of promoter activities, the basal expression level decreased. This region may therefore contain sequences with which auxiliary transcriptional regulators interact to increase the overall amount of expression. The promoter regions of $N b E A S 3$ and NbEAS4 have two GCC-box like motifs (Supplementary Figure 1). NbEAS3 and NbEAS4 are the most highly expressed homologs of NbEAS (Figure 3), thus this second GCC-box-like sequence might be responsible for the stronger INF1-induced gene expression.

In control samples we observed a constant expression of MVA pathway genes, which was to be expected, since besides producing the capsidiol precursor FPP, the pathway is of central importance for a large variety of isoprenoid compounds. A quick and ample production of capsidiol would require the MVA pathway to step up production, which appears to be the case, as INF1 elicitation could be shown to up-regulate several MVA genes (Figure 4). Despite this, the GCC-box-like motif was not found in the promoters of MVA genes, not even NbHMGR2, which was contained in cluster 14 with several NbEAS genes. This is somewhat surprising, since the upregulation of the MVA pathway during pathogenesis is typically not observed in plants that do not produce terpenoid defense compounds, and we would therefore assume that this regulatory mechanism had co-evolved with terpenoid phytoalexin synthesis. An explanation of why the GCC-box motif is absent in MVA promoters is purely speculative at this point, but one reason could be that terpenoid phytoalexins are preferentially produced against necro- and hemibiotrophic pathogens, and the MVA pathway may need to be tightly regulated during defense against biotic pathogens as well. Ishihama et al. (2011) has reported that $N b H M G R 2$ is under the control of a defense-related transcription factor, 
482

483

484

485

486

487

488

489

490

491

492

493

494

495

496

497

498

499

500

501

502

503

504

505

506

507

508

509

510

511

512

513

514

515

516

517

518

519

520

521

522

523

524

525

526

527

528

529

530

NbWRKY8, which targets W-boxes, but further investigation of the activation mechanism of genes in the MVA pathway would be an interesting subject of future research.

AP2/ERF family is a conserved group of plant transcription factors, being defined by a central AP2/ERF domain consisting of approx. 60 amino acid residues, which bind to cis-element targets (Licausi et al., 2013). Although they are called "ethylene-responsive factors" due to how they were first discovered (Ohme-Tagaki and Shinshi, 1995), ERFs are involved in the regulation of diverse phenomena, including development, morphogenesis, and biotic/abiotic stress responses mediated via different plant hormones. For instance, Arabidopsis PUCHI (belonging to subfamily ERF-VIII) is involved in lateral root initiation and development via auxin-mediated signaling (Hirota et al., 2007), while CRFs (cytokinin response factors, belonging to ERF-VI) are induced by cytokinin, which functions in the regulation of root growth, embryo development, leaf senescence, and hypocotyl elongation (Kim, 2016). In this study, we listed all genes predicted to encode AP2/ERF transcription factors in the $N$. benthamiana genome to identify the transcription factors that directly control phytoalexin production via GCC-box-like sequences in NbEAS1 and NbEAS4 promoters. A total of $283 E R F$ genes were found, of which 43 (15.2\%) were assigned to the cluster for INF1-inducible genes (Supplementary Table 12). Given that INF1 treatment enhanced the production of ethylene within 9 h (Supplementary Figure 2), a substantial number of ERF gene groups were shown not to be induced by ethylene.

The functional analysis of two NbERFs (both belonging to ERF-IX), whose expression was significantly increased by INF1 treatment, indicated that NbERF-IX-33 is an essential transcription factor for the INF1-induced production of capsidiol and the resistance to P. infestans (Figure 8). Gene silencing of $N b E R F-I X-16$ may reduce the capsidiol production (this could not be shown to be statistically significant), and the $N b E R F-I X-16$-silenced plant were slightly more susceptible to $P$. infestans (Figure 8). The reduction in disease resistance and capsidiol production in NbEIN2-silenced plants was more pronounced than in NbERF-IX-33-silenced plants, suggesting that EIN2 may act upstream of NbERF regulation, affecting multiple NbERFs involved in the induction of phytoalexin production.

In this study, we reported that NbERF-IX-33a binds to the GCC-box like motif in the promoter region of NbEAS4, which is supported by many studies that have shown ERFs that bind to the GCC box. Four ERFs were originally isolated from tobacco as transcription factors that directly bind to the GCC box, the conserved motif found in the promoter region of ethylene-inducible $P R$ genes (OhmeTakagi and Shinshi, 1995). In Arabidopsis, ORA59 and ERF1/ERF92 (subfamily IX) have been shown to activate jasmonic acid- and ethylene- mediated expression of the defensin gene AtPDF1.2 by directly binding to two GCC boxes in its promoter region (Zarei et al., 2011), while AtERF3 and AtERF4 can downregulate their target gene via a GCC box (Fujimoto et al., 2000). In rice, OsERF83 positively regulates the disease resistance against rice blast pathogen by upregulating $P R$ genes, whereas OsERF922 activates abscisic acid biosynthesis-related genes which has a negative effect on the disease resistance (Liu et al., 2012; Tezuka et al. 2019). Tobacco ERF189 and four related ERFs (subfamily IX) can bind to a GCC-box in the promoter region of PMT (putrescine $\mathrm{N}$ methyltransferase) for jasmonate-inducible nicotine synthesis, and all enzyme genes for nicotine production are under the control of ERF189 (Shoji et al., 2010). Wheat ERF transcription factor TaERF3 is involved in salt and drought tolerance via the GCC boxes in stress-related genes (Rong et al., 2014). Expression of genes for the production of anti-insect steroidal glycoalkaloids (SGA) in tomato is under the control of jasmonate-responsive ERF, JRE4, which bind to GCC box-like elements in the promoter of SGA biosynthetic genes (Thagun et al., 2016). These reports indicate that GCC-box and ERF combinations are involved in the regulation of diverse phenomena in different plant species.

The synthesis of bioactive compounds requires a high degree of coordination, especially in cases where other pathways rely on the same substrate (such as FPP), and where its depletion can therefore 
531

532

533

534

535

536

537

538

539

540

541

542

543

544

545

546

547

548

549

550

551

552

553

554

555

556

557

558

559

560

561

562

563

564

565

566

567

568

569

570

571

572

573

574

575

576

577

578

have widespread negative effects. This poses a problem during the evolution of a novel pathway, since it must assure that other pathways are not disrupted. Shoji and Yuan (2021) suggest that the diversified combinations of a collection of enzyme genes under the control of the same transcriptional factors can lead to the invention of a new metabolic pathway in different plant species. In addition to the promoters of genes for $\mathrm{NbEAS}$ and $\mathrm{NbEAH}$, which consist of a short metabolic pathway for capsidiol production, the GCC-box like motifs are also conserved in the promoter region of $N b A B C G 1$ and 2 genes, which is involved in the secretion of capsidiol at the sites of pathogens attack (Shibata et al., 2016; Rin et al., 2017). Thus, this theory may be valid beyond the enzyme genes of the metabolic pathways. Rishitin, a sesquiterpenoid phytoalexin produced by potato and tomato, is expected to be produced by a more complex metabolic pathway compared with capsidiol, but the pathway for rishitin production is still largely unknown. Since the two primary rishitinproducing enzymes also share a GCC box-like promoter sequence (Takemoto et al., 2018), the similarity of expression patterns and the commonality of the regulating transcription factors may provide a clue to the elucidation of this yet unknown biosynthetic pathway.

\section{Author contributions}

DT designed the research. SI, MF, AT-K, HM, AA, AT and DT conducted the experiments. SI, MF, MC, AA and DT analyzed data, A Tanaka, IS, SC, KK, MO and DT supervised the experiments. SI and DT wrote the manuscript. MC and DT edited the manuscript. SI, MC and DT contributed to the discussion and interpretation of the results.

\section{Funding}

This work was supported by a Grant-in-Aid for Scientific Research (B) (17H03771 and 20H02985) to DT and a Grant-in-Aid for JSPS Fellows (21J14198) to SI from the Japan Society for the Promotion of Science.

\section{Acknowledgments}

We thank Prof. David C. Baulcombe (University of Cambridge, USA) for providing pTV00 and pBINTRA6 vectors, Prof. Sophien Kamoun (The Sainsbury Laboratory, UK) for the pFB53 vector, and Prof. Gregory B. Martin (Cornell University, USA) for the access to the N. benthamiana genome database. We also thank Dr. David Jones (The Australian National University, Australia) for $N$. benthamiana seeds, Ms. Kayo Shirai (Hokkaido Central Agricultural Experiment Station, Japan), and Dr. Seishi Akino (Hokkaido University, Japan) for providing P. infestans isolate 08YD1. We also thank Dr. Kenji Asano and Mr. Seiji Tamiya (National Agricultural Research Center for Hokkaido Region, Japan) and Mr. Yasuki Tahara (Nagoya University, Japan) for providing tubers of potato cultivars.

\section{REFERENCES}

Blein, J.-P., Coutos-Thevenot, P., Marion, D., and Ponchet, M. (2002). From elicitins to lipid-transfer proteins: a new insight in cell signalling involved in plant defence mechanisms. Trends Plant Sci. 7, 293-296.

Boissy, G., de La Fortelle, E., Kahn, R., Huet, J.-C., Bricogne, G., Pernollet, J.-C., and Brunie, S. (1996). Crystal structure of a fungal elicitor secreted by Phytophthora cryptogea, a member of a novel class of plant necrotic proteins. Structure 4, 1429-1439. 
603

604

605

606

607

608

609

610

611

612

613

614

615

616

617

618

619

620

621

622

623

624

625

Bombarely, A., Rosli, H.G., Vrebalov, J., Moffett, P., Mueller, L.A., and Martin, G.B. (2012). A draft genome sequence of Nicotiana benthamiana to enhance molecular plant-microbe biology research. Mol. Plant-Microbe Interact. 25, 1523-1530.

Bonnet, P., Bourdon, E., Ponchet, M., Blein, J.P., and Ricci, P. (1996). Acquired resistance triggered by elicitins in tobacco and other plants. Eur. J. Plant Pathol. 102, 181-192.

Cabral, A., Stassen, J.H., Seidl, M.F., Bautor, J., Parker, J.E., and van den Ackerveken, G. (2011). Identification of Hyaloperonospora arabidopsidis transcript sequences expressed during infection reveals isolate-specific effectors. PLoS One 6, e19328.

Cañizares, M.C., Lozano-Durán, R., Canto, T., Bejarano, E.R., Bisaro, D.M., Navas-Castillo, J., and Moriones, E. (2013). Effects of the crinivirus coat protein-interacting plant protein SAHH on posttranscriptional RNA silencing and its suppression. Mol. Plant-Microbe Interact. 26, 1004-1015.

Chai, H.B., and Doke, N. (1987). Activation of the potential of potato leaf tissue to react hypersensitively to Phytophthora infestans by cystospore germination fluid and the enhancement of the potential by calcium ion. Physiol. Mol. Plant Pathol. 30, 27-37.

Colas, V., Conrod, S., Venard, P., Keller, H., Ricci, P., and Panabières, F. (2001). Elicitin genes expressed in vitro by certain tobacco isolates of Phytophthora parasitica are down regulated during compatible interactions. Mol. Plant-Microbe Interact. 14, 326-335.

Denancé, N., Sánchez-Vallet, A., Goffner, D., and Molina, A. (2013). Disease resistance or growth: the role of plant hormones in balancing immune responses and fitness costs. Front. Plant Sci. 4, 155.

Du, J., Verzaux, E., Chaparro-Garcia, A., Bijsterbosch, G., Keizer, L.C., Zhou, J., Liebrand, T.W., Xie, C., Govers, F., Robatzek, S., van der Vossen, E.A., Jacobsen, E., Visser, R.G., Kamoun, S., and Vleeshouwers, V.G. (2015). Elicitin recognition confers enhanced resistance to Phytophthora infestans in potato. Nat. Plants 1, 15034.

Fernandez-Pozo, N., Rosli, H.G., Martin, G.B., and Mueller, L.A. (2015). The SGN VIGS tool: userfriendly software to design virus- induced gene silencing (VIGS) constructs for functional genomics. Mol. Plant 8, 486-488.

Fujimoto, S.Y., Ohta, M., Usui, A., Shinshi, H., and Ohme-Takagi, M. (2000). Arabidopsis ethyleneresponsive element binding factors act as transcriptional activators or repressors of GCC boxmediated gene expression. Plant Cell 12, 393-404.

Glazebrook, J. (2005). Contrasting mechanisms of defense against biotrophic and necrotrophic pathogens. Annu. Rev. Phytopathol. 43, 205-227.

Goodin, M.M., Zaitlin, D., Naidu, R.A., and Lommel, S.A. (2008). Nicotiana benthamiana: its history and future as a model for plant-pathogen interactions. Mol. Plant-Microbe Interact. 21, 1015-1026. 
646

647

648

649

650

651

652

653

654

655

656

657

658

659

660

661

662

663

664

665

666

667

668

669

670

671

672

Haverkort, A.J., Boonekamp, P.M., Hutten, R., Jacobsen, E., Lotz, L.A.P., Kessel, G.J.T., Visser, R.G.F., and van der Vossen, E.A.G. (2008). Societal costs of late blight in potato and prospects of durable resistance through cisgenic modification. Potato Res. 51, 47-57.

Hendrix, J.W. (1970). Sterols in growth and reproduction of fungi. Annu. Rev. Phytopathol. 8, 111130.

Hirota, A., Kato, T., Fukaki, H., Aida, M., and Tasaka, M. (2007). The auxin-regulated AP2/EREBP gene PUCHI is required for morphogenesis in the early lateral root primordium of Arabidopsis. Plant Cell 19, 2156-2168.

Ishihama, N., Yamada, R., Yoshioka, M., Katou, S., and Yoshioka, H. (2011). Phosphorylation of the Nicotiana benthamiana WRKY8 transcription factor by MAPK functions in the defense response. Plant Cell 23, 1153-1170.

Ismayil, A., Haxim, Y., Wang, Y., Li, H., Qian, L., Han, T., Chen, T., Jia, Q., Yihao Liu, A., Zhu, S., Deng, H., Gorovits, R., Hong, Y., Hanley-Bowdoin, L., and Liu, Y. (2018). Cotton Leaf Curl Multan virus $\mathrm{C} 4$ protein suppresses both transcriptional and post-transcriptional gene silencing by interacting with SAM synthetase. PLoS Pathog. 14, e1007282.

Jiang, R.H.Y., Tyler, B.M., Whisson, S.C., Hardham, A.R. and Govers, F. (2006). Ancient origin of elicitin gene clusters in Phytophthora genomes. Mol. Biol. Evol. 23, 338-351.

Kamoun, S., van West, P., deJong, A.J., deGroot, K.E., Vleeshouwers, V.G., and Govers, F. (1997). A gene encoding a protein elicitor of Phytophthora infestans is down-regulated during infection of potato. Mol. Plant-Microbe Interact. 10, 13-20.

Kamoun, S., van West, P., Vleeshouwers, V.G., de Groot, K.E., and Govers, F. (1998). Resistance of Nicotiana benthamiana to Phytophthora infestans is mediated by the recognition of the elicitor protein INF1. Plant Cell 10, 1413-1426.

Kim, J. (2016). Cytokinin response factors gating environmental signals and hormones. Trends Plant Sci. 21, 993-996.

Kim, D., Paggi, J.M., Park,, C., Bennett, C., and Salzberg, S.L. (2019). Graph-based genome alignment and genotyping with HISAT2 and HISAT-genotype. Nat. Biotechnol. 37, 907-915.

Kovaka, S., Zimin, A.V., Pertea, G.M., Razaghi, R., Salzberg, S.L., Pertea, M. (2019). Transcriptome assembly from long-read RNA-seq alignments with StringTie2. Genome Biol. 20, 278.

Levine, A., Tenhaken, R., Dixon, R., and Lamb, C. (1994). $\mathrm{H}_{2} \mathrm{O}_{2}$ from the oxidative burst orchestrates the plant hypersensitive disease resistance response. Cell 79, 583-593.

Licausi, F., Ohme-Takagi, M., and Perata, P. (2013). APETALA2/Ethylene Responsive Factor (AP2/ERF) transcription factors: mediators of stress responses and developmental programs. New Phytol. 199, 639-649. 
673

674

675

676

677

678

679

680

681

682

683

684

685

686

687

688

689

690

691

692

693

694

695

696

697

698

699

700

701

702

703

704

705

706

707

708

709

710

711

712

713

714

715

716

717

718

719

720

Liu, D., Chen, X., Liu, J., Ye, J., and Guo, Z. (2012). The rice ERF transcription factor OsERF922 negatively regulates resistance to Magnaporthe oryzae and salt tolerance. J. Exp Bot. 63, 38993911.

Love, M.I., Huber, W., and Anders, S. (2014). Moderated estimation of fold change and dispersion for RNA-seq data with DESeq2. Genome Biol. 15, 550

Maldonado-Bonilla, L.D., Betancourt-Jiménez, M., and Lozoya-Gloria, E. (2008). Local and systemic gene expression of sesquiterpene phytoalexin biosynthetic enzymes in plant leaves. Eur. J. Plant Pathol. 121, 439-449.

Martin, M. (2011). Cutadapt removes adapter sequences from high-throughput sequencing reads. EMBnet J. 17, 10-12.

Matsukawa, M., Shibata, Y., Ohtsu, M., Mizutani, A., Mori, H., Wang, P., Ojika, M., Kawakita, K., and Takemoto, D. (2013). Nicotiana benthamiana calreticulin 3a is required for the ethylenemediated production of phytoalexins and disease resistance against oomycete pathogen Phytophthora infestans. Mol. Plant-Microbe Interact. 26, 880-892.

Mikes, V., Milat, M.-L., Ponchet, M., Panabières, F., Ricci, P., and Blein, J.-P. (1998). Elicitins, proteinaceous elicitors of plant defense, are a new class of sterol carrier proteins. Biochem. Biophys. Res. Commun. 245, 133-139.

Milat, M.-L., Ricci, P., Bonnet, P., and Blein, J.-P. (1991). Capsidiol and ethylene production by tobacco cells in response to cryptogein, an elicitor from Phytophthora cryptogea. Phytochemistry 30, 2171-2173.

Mizuno, Y., Ohtsu, M., Shibata, Y., Tanaka, A., Camagna, M., Ojika, M., Mori, H., Sato, I., Chiba, S., Kawakita, K., and Takemoto, D. (2019a). Nicotiana benthamiana RanBP1-1 is involved in the induction of disease resistance via regulation of nuclear-cytoplasmic transport of small GTPase Ran. Front Plant Sci. 10, 222.

Mizuno, Y., Imano, S., Camagna, M., Suzuki, T., Tanaka, A., Sato, I., Chiba, S., Kawakita, K., and Takemoto D. (2019b). Nicotiana benthamiana exportin 1 is required for elicitor $\square$ induced phytoalexin production, cell death induction, and resistance against potato late blight pathogen Phytophthora infestans. J. Gen. Plant Pathol. 85, 347-355.

Monjil, M.S., Kato, H., Matsuda, K., Suziki, N., Tenhiro, S., Camagna, M., Suzuki, T., Tanaka, A., Terauchi, R., Sato, I., Chiba, S., Kawakita, K., Ojika, M., and Takemoto D. (2021). Two structurally different oomycete MAMPs induce distinctive plant immune responses. bioRxiv DOI: 10.1101/2021.10.22.465218

Müller, M., and Munné-Bosch, S. (2015). Ethylene Response Factors: A key regulatory hub in hormone and stress signaling. Plant Physiol. 169, 32-41.

Nakano, T., Suzuki, K., Fujimura, T., and Shinshi, H. (2006). Genome-wide analysis of the ERF gene family in Arabidopsis and rice. Plant Physiol. 140, 411-432. 
731

732

733

734

735

736

737

738

739

740

741

742

743

744

745

746

747

748

749

750

751

752

753

754

755

756

757

758

759

760

761

762

763

764

765

766

767

768

Ohme-Takagi, M., and Shinshi, H. (1995). Ethylene-inducible DNA binding proteins that interact with an ethylene-responsive element. Plant Cell 7, 173-182.

Ohtsu, M., Shibata, Y., Ojika, M., Tamura, K., Hara-Nishimura, I., Mori, H., Kawakita, K., and Takemoto, D. (2014). Nucleoporin 75 is involved in the ethylene-mediated production of phytoalexin for the resistance of Nicotiana benthamiana to Phytophthora infestans. Mol. PlantMicrobe Interact. 27, 1318-1330.

Ranf, S. (2017). Sensing of molecular patterns through cell surface immune receptors. Curr. Opin. Plant Biol. 38, 68-77.

Ratcliff, F., Martin-Hernandez, A.M., and Baulcombe, D.C. (2001). Tobacco rattle virus as a vector for analysis of gene function by silencing. Plant J. 25, 237-245.

Ricci, P., Trentin, F., Bonnet, P., Venard, P., Mouton-Perronnet, F., and Bruneteau, M. (1992). Differential production of parasiticein, an elicitor of necrosis and resistance in tobacco, by isolates of Phytophthora parasitica. Plant Pathol. 41, 298-307.

Rin, S., Mizuno, Y., Shibata, Y., Fushimi, M., Katou, S., Sato, I., Chiba, S., Kawakita, K., and Takemoto, D. (2017). EIN2-mediated signaling is involved in pre-invasion defense in Nicotiana benthamiana against potato late blight pathogen, Phytophthora infestans. Plant Signal. Behav. 12, e1300733.

Rin, S., Imano, S., Camagna, M., Suzuki, T., Tanaka, A., Sato, I., Chiba, S., Kawakita, K., and Takemoto, D. (2020). Expression profiles of genes for enzymes involved in capsidiol production in Nicotiana benthamiana. J. Gen. Plant Pathol. 86, 340-349.

Rong, W., Qi, L., Wang, A., Ye, X., Du, L., Liang, H., Xin, Z., and Zhang, Z. (2014). The ERF transcription factor TaERF3 promotes tolerance to salt and drought stresses in wheat. Plant Biotechnol J. 12:468-479.

Saitou, N., and Nei, M. (1987). The neighbor-joining method: a new method for reconstructing phylogenetic trees. Mol. Biol. Evol. 4, 406-425.

Schenk, P.M., Kazan, K., Wilson, I., Anderson, J.P., Richmond, T., Somerville, S.C., and Manners, J.M. (2000). Coordinated plant defense responses in Arabidopsis revealed by microarray analysis. Proc. Natl. Acad. Sci. USA 97, 11655-11660.

Shibata, Y., Kawakita, K., and Takemoto, D. (2010). Age-related resistance of Nicotiana benthamiana against hemibiotrophic pathogen Phytophthora infestans requires both ethylene- and salicylic acid-mediated signaling pathways. Mol. Plant-Microbe Interact. 23, 1130-1142.

Shibata, Y., Kawakita, K., and Takemoto, D. (2011). SGT1 and HSP90 are essential for age-related non-host resistance of Nicotiana benthamiana against the oomycete pathogen Phytophthora infestans. Physiol. Mol. Plant Pathol. 75, 120-128.

Shibata, Y., Ojika, M., Sugiyama, A., Yazaki, K., Jones, D.A., Kawakita, K., and Takemoto, D. (2016). The full-size ABCG transporters Nb-ABCG1 and Nb-ABCG2 function in pre- and 
postinvasion defense against Phytophthora infestans in Nicotiana benthamiana. Plant Cell 28, 1163-1181.

Shoji, T., Kajikawa, M., and Hashimoto, T. (2010). Clustered transcription factor genes regulate nicotine biosynthesis in tobacco. Plant Cell 22, 3390-3409.

Shoji, T., and Yuan, L. (2021). ERF gene clusters: working together to regulate metabolism. Trends Plant Sci. 26, 23-32.

Takemoto, D., Hardham, A.R., and Jones, D.A. (2005). Differences in cell death induction by Phytophthora elicitins are determined by signal components downstream of MAP kinase kinase in different species of Nicotiana and cultivars of Brassica rapa and Raphanus sativus. Plant Physiol. $138,1491-504$.

Takemoto, D., Shibata, Y., Ojika, M., Mizuno, Y., Imano, S., Ohtsu, M., Sato, I., Chiba, S., Kawakita, K., Rin, S., and Camagna, M. (2018). Resistance to Phytophthora infestans: exploring genes required for disease resistance in Solanaceae plants. J. Gen. Plant Pathol. 84, 312-320.

Tezuka, D., Kawamata, A., Kato, H., Saburi, W., Mori, H., and Imai, R. (2019). The rice ethylene response factor OsERF83 positively regulates disease resistance to Magnaporthe oryzae. Plant Physiol Biochem. 2019 Feb;135:263-271.

Thagun, C., Imanishi, S., Kudo, T., Nakabayashi, R., Ohyama, K., Mori, T., Kawamoto, K., Nakamura, Y., Katayama, M., Nonaka, S., Matsukura, C., Yano, K., Ezura, H., Saito, K., Hashimoto, T., Shoji, T. (2016). Jasmonate-responsive ERF transcription factors regulate steroidal glycoalkaloid biosynthesis in tomato. Plant Cell Physiol. 57, 961-975.

Thompson, J.D., Higgins, D.G., and Gibson, T.J. (1994). CLUSTAL W: improving the sensitivity of progressive multiple sequence alignment through sequence weighting, position-specific gap penalties and weight matrix choice. Nucleic Acids Res. 22, 4673-4680.

Todd, A.T., Liu, E., Polvi, S.L., Pammett, R.T., and Page, J.E. (2010). A functional genomics screen identifies diverse transcription factors that regulate alkaloid biosynthesis in Nicotiana benthamiana. Plant J. 62, 589-600.

Tyler, B.M. (2002). Molecular basis of recognition between Phytophthora pathogens and their hosts. Annu. Rev. Phytopathol. 40, 137-167.

Winter, D., Vinegar, B., Nahal, H., Ammar, R., Wilson, G.V., and Provart, N.J. (2007). An "Electronic Fluorescent Pictograph" browser for exploring and analyzing large-scale biological data sets. PLoS One 2, e718.

Yamaguchi-Shinozaki K, and Shinozaki K. (1994). A novel cis-acting element in an Arabidopsis gene is involved in responsiveness to drought, low-temperature, or high-salt stress. Plant Cell 6, 251-64.

Yu, J., Chai, C., Ai, G., Jia, Y., Liu, W., Zhang, X., Bai, T., and Dou, D. (2020). A Nicotiana benthamiana AP2/ERF transcription factor confers resistance to Phytophthora parasitica. Phytopathol Res. 2, 4. 
818

819

820

821

822

823

824

825

826

827

828

829

830

831

832

833

834

835

836

837

838

839

840

841

842

843

844

845

846

847

848

849

850

851

852

853

854

855

856

857

858

859

860

861

862

863

864

Yuan, M., Jiang, Z., Bi, G., Nomura, K., Liu, M., Wang, Y., Cai, B., Zhou, J.M., He, S.Y., and Xin, X.F. (2021). Pattern-recognition receptors are required for NLR-mediated plant immunity. Nature 592, 105-109.

Zarei, A., Körbes, A.P., Younessi, P., Montiel, G., Champion, A., and Memelink, J. (2011). Two GCC boxes and AP2/ERF-domain transcription factor ORA59 in jasmonate/ethylene-mediated activation of the PDF1.2 promoter in Arabidopsis. Plant Mol. Biol. 75, 321-331.

\section{FIGURE LEGENDS}

FIGURE 1 | Induction of defense responses of Nicotiana benthamiana leaves treated with INF1, a secretory protein derived from Phytophthora infestans. (A) INF1-induced reactive oxygen species (ROS) production in $N$. benthamiana. Leaf discs were placed in water $\left(\mathrm{H}_{2} \mathrm{O}\right)$ or $150 \mathrm{nM}$ INF1 solution containing L-012, and L-012 mediated chemiluminescence was measured. Data are means \pm standard error $(\mathrm{SE})(\mathrm{n}=8)$. (B) Leaves of $N$. benthamiana were treated with water, or $150 \mathrm{nM}$ INF1 by infiltration and ROS production was detected at indicated time after the treatment. Data are means \pm standard error $(\mathrm{SE})(\mathrm{n}=6)$. $(\mathrm{C})$ Phytoalexins were extracted at the indicated time after water $(\mathrm{H})$ or 150 nM INF1 (I) treatment and quantified by LC/MS. Data are means \pm SE $(\mathrm{n}=3)$. (D) Leaves of $N$. benthamiana were treated with water or $150 \mathrm{nM} \mathrm{INF1,} \mathrm{and} \mathrm{electrolyte} \mathrm{leakage} \mathrm{was} \mathrm{quantified} \mathrm{at} 24 \mathrm{~h}$ after INF1 treatment. Data are means \pm SE $(n=6)$. Photographs were taken $72 \mathrm{~h}$ after the treatment. Data marked with asterisks are significantly different from control as assessed by the two-tailed Student's $t$-test: $* * P<0.01$.

FIGURE 2 | Clustering of 57,140 Nicotiana benthamiana genes based on their expression profiles in leaves treated with water or $150 \mathrm{nM}$ INF1. 14,390 (25.2\%) of genes were not assigned to any cluster because no or significantly lower expression was detected. See method for detailed procedure of the gene clustering. Graphs shown are average expression profiles of 100 genes (selected based on their high average FPKM value) from each cluster. Data are means \pm SE $(n=100)$. Clusters for INF1 induced genes are shown in red letters.

FIGURE 3 | The time course of expression of Nicotiana benthamiana genes encoding dedicated enzymes for phytoalexin production. The gene expression (FPKM value) was determined by RNAseq analysis of $N$. benthamiana leaves treated with water or $150 \mathrm{nM}$ INF1 for $0 \mathrm{~h}, 3 \mathrm{~h}, 6 \mathrm{~h}, 12 \mathrm{~h}$ and $24 \mathrm{~h}$. Data are mean $\pm \operatorname{SE}(n=3)$. Asterisks indicate a significant difference from the control (watertreated) as assessed by two-tailed Student's $t$-test, ${ }^{* *} P<0.01$. EAS 5-epi-aristolochene synthase, EAH 5-epi-aristolochene-1,3-dihydroxylase

FIGURE 4 | The time course of expression of Nicotiana benthamiana genes encoding enzymes in the mevalonate pathway. Gene expression (FPKM value) was determined by RNA-seq analysis of $N$. benthamiana leaves treated with water $\left(\mathrm{H}_{2} \mathrm{O}\right)$ or $150 \mathrm{nM}$ INF1 for $3 \mathrm{~h}, 6 \mathrm{~h}, 12 \mathrm{~h}$ and $24 \mathrm{~h}$. Data are means $\pm \mathrm{SE}(\mathrm{n}=3)$. Data marked with asterisks are significantly different from control as assessed by the two-tailed Student's $t$-test: ${ }^{*} P<0.01 * P<0.05$. ACAT, Acetoacetyl-CoA thiolase; $H M G S$, Hydroxymethylglutaryl-CoA synthase; HMGR, Hydroxymethylglutaryl-CoA-reductase; $M V K$, Mevalonate-5-kinase; PMVK, Phosphomevalonate kinase; MVD, Mevalonate-5-pyrophosphate decarboxylase; IPPI, Isopentenyl pyrophosphate isomerase; FPPS, Farnesyl pyrophosphate synthase. 
FIGURE 5 | Identification of cis-element for INF1-induced expression of $N b E A S$ genes. (A) Expression of GFP gene under the control of the indicated length of $N b E A S 1$ and NbEAS4 promoter (P_EAS). Total RNA was isolated from $N$. benthamiana leaves $48 \mathrm{~h}$ after co-inoculation with $A$. tumefaciens strains containing expression vectors for $\mathrm{P}_{-} E A S: G F P$, INF1 or control (C) vector, and truncated GUS (tGUS) gene under the control of $35 \mathrm{~S}$ promoter as internal standard. Expression of the GFP gene was assessed by qRT-PCR with GFP primers and values were normalized to the expression of $t G U S$. Means $\pm \mathrm{SE}(\mathrm{n}=3)$. Data marked with asterisks are significantly different as assessed by the two-tailed Student's $t$-test: $* P<0.05$. (B) Alignment of -260 to -230 of $N b E A S 1$ promoter and -230 to -200 of NbEAS4 promoter. GCC box-like motifs were shown in red letters. Mutations introduced in NbEAS4 (TT) were shown below the alignment by boxes. (C) Expression of GFP gene under the control of 230 bp of wild type or mutated (TT) NbEAS4 promoter. (Left) Fluorescence micrographs of $N$. benthamiana leave $48 \mathrm{~h}$ after A. tumefaciens inoculation containing indicated expression vectors. Bars $=500 \mu \mathrm{m}$. (Right) Total RNA was isolated from $N$. benthamiana leaves $48 \mathrm{~h}$ after co-inoculation with $A$. tumefaciens strains containing expression vectors. Expression of the GFP gene was assessed by qRT-PCR with GFP primers and values were normalized to the expression of $t G U S$. Means $\pm \mathrm{SE}(\mathrm{n}=3)$. Data marked with asterisks are significantly different as assessed by the two-tailed Student's $t$-test: $* P<0.05$.

FIGURE 6 | Expression profiles of Nicotiana benthamiana genes encoding enzymes for ethylene production. The gene expression (FPKM value) was determined by RNA-seq analysis of $N$. benthamiana leaves treated with water and $150 \mathrm{nM}$ INF1 for $0 \mathrm{~h}, 3 \mathrm{~h}, 6 \mathrm{~h}, 12 \mathrm{~h}, 24 \mathrm{~h}$. Data are means $\pm \mathrm{SE}(\mathrm{n}=3)$. Data marked with asterisks are significantly different from control as assessed by the two-tailed Student's $t$-test: $* * P<0.01, * P<0.05$. CGS, Cystathionine gamma-synthase; SAMS, S-adenosylmethionine synthetase; SAHH, S-adenosyl homocysteine hydrolase; ACS, Aminocyclopropane carboxylate synthase; ACO, Aminocyclopropane carboxylate oxidase.

FIGURE 7 | An unrooted phylogenetic tree of AP2/ERF transcription factors form Nicotiana benthamiana and Arabidopsis thaliana. The deduced amino acid sequences of AP2/ERF transcription factors were aligned by ClustalW (Thompson et al., 1994), and the phylogenetic tree was constructed using the neighbor-joining (NJ) method (Saitou and Nei, 1987). Classification of AP2/ERF transcription factors by Nakano et al. (2006) are indicated. The cluster numbers of INF1-inducible genes are shown in red letters.

899

900

FIGURE 8 | (A) Expression profiles of Nicotiana benthamiana genes encoding two selected ERF transcription factors. Gene expression (FPKM value) was determined by RNA-seq analysis of $N$. benthamiana leaves treated with water $\left(\mathrm{H}_{2} \mathrm{O}\right)$ or $150 \mathrm{nM} \mathrm{INF} 1$. Data are means $\pm \mathrm{SE}(\mathrm{n}=3)$. Data marked with asterisks are significantly different from control (water-treated). $* * P<0.01$. (B) $N$. benthamiana were inoculated with TRV, TRV:ERF-IX-33 or TRV:ERF-IX-16 and the right side of leaves of control or gene-silenced plants were inoculated with $P$. infestans. The appearance of disease symptoms was categorized into 5 classes according to the severity of disease symptoms. 0 , no visible symptom; 1 , small wilted spots in inoculated area; 2 , browning $<50 \%$ of the inoculated side of leaf; 3 , browning $>50 \%$ of the inoculated side of leaf; 4 , development of disease symptoms over central leaf vein. Plot showing percentage of $N$. benthamiana leaves with disease symptom severities represented in the five classes as shown in the upper panels, for leaves of control and gene-silenced plants inoculated with $P$. infestans from 3 to 5 days post-inoculation (dpi). At least 50 leaves from each control and gene-silenced plant were scored. Data marked with asterisks are significantly different from control as assessed by one-tailed Mann-Whitney $U$ tests: ${ }^{*} P<0.05$. (C) Production of 
phytoalexin in TRV-inoculated or gene-silenced $N$. benthamiana. Leaves were harvested 24 hours after $150 \mathrm{nM}$ INF1 treatment and extracted phytoalexins were detected by HPLC. Data are means \pm SE $(\mathrm{n}=4)$. Data marked with asterisks are significantly different from control. $* * P<0.01$. (D) Binding of NbERF-IX33 to the NbEAS4 promoter. MBP (maltose-binding protein) or MBP-ERF-IX-

918

919 33 a was incubated with NbEAS4 promoter fragments. Samples were separated in a non-denaturing polyacrylamide gel. The gel was stained with SYBR Green EMSA stain.

\section{SUPPLEMENTAL DATA}

SUPPLEMENTARY FIGURE 1 | Nucleotide sequence of the promoter region of $N b E A S$ and $\mathrm{NbEAH}$ genes. The 7-bp sequences for the GCC box-like motifs are shown in red letters, the 6-bp sequences for the W-box motifs are shown in blue letters, and the start codon of $N b E A S$ and $N b E A H$ are shown in orange letters. Note that the promoter sequence for $N b E A S 10$ ( -350 to -237$)$ is not available.

SUPPLEMENTARY FIGURE 2 | (A) Accumulation of ethylene in N. benthamiana leaves treated with INF1. Leaves of control (TRV) or $N b A C O$-silenced (TRV:ACO) were treated with water $\left(\mathrm{H}_{2} \mathrm{O}\right)$, or $150 \mathrm{nM}$ INF1 and the amount of ethylene produced was measured by gas chromatography. Data are means \pm SE $(n=4)$. (B) $N$. benthamiana were inoculated with TRV, TRV:EIN2 or TRV:ACO and leaves of control or $\mathrm{NbACO}$-silenced plants were inoculated with $P$. infestans. The appearance of disease symptoms was categorized into 3 classes according to the severity of disease symptoms. 0 , no visible symptom; 1 , small wilted spots in inoculated area; 2 , browning $>50 \%$ of the inoculated side of the leaf. Plot showing percentage of $N$. benthamiana leaves with disease symptom severities represented in the three classes as shown in the upper panels, for leaves of control and gene-silenced plants inoculated with $P$. infestans at 7days post-inoculation (dpi). At least 12 leaves from each control and gene-silenced plant were scored. Data marked with asterisks are significantly different from control as assessed by one-tailed Mann-Whitney U tests: $* P<0.05$.

SUPPLEMENTARY FIGURE 3 | Expression and purification of NbERF-IX-33a proteins in Escherichia coli. E. coli with pMAL-c5x or pMAL-c5x containing NbERF-IX-33 gene were cultured in LB medium with IPTG for the induction of protein expression. Cultured E. coli cells were harvested 5 hours after IPTG treatment and MBP (maltose-binding protein) or MBP- NbERF-IX-33a were purified using amylose resin. Eluted fractions were separated by SDS-PAGE and stained with $\mathrm{CBB}$. Protein size markers are shown in $\mathrm{kDa}$. The concentrations of the purified proteins were adjusted and used for the experiments in Figure 8D.

Supplementary Table 1 Plasmids used in this study.

Supplementary Table 2 Primers used in this study.

Supplementary Table 3 Nicotiana benthamiana genes categorized in cluster 2 .

Supplementary Table 4 Nicotiana benthamiana genes categorized in cluster 4 .

Supplementary Table 5 Nicotiana benthamiana genes categorized in cluster 10 . 
Supplementary Table 7 Nicotiana benthamiana genes categorized in cluster 16.

966

Supplementary Table 8 Predicted Nicotiana benthamiana genes for enzymes specifically involved

967

968 in the production of capsidiol.

969

970

971

972

973

Supplementary Table 9 Predicted genes for enzymes in the mevalonate pathway and farnesylpyrophosphate synthase in Nicotiana benthamiana.

976

Supplementary Table 10 Predicted genes for enzymes involved in the methionine cycle and ethylene production in Nicotiana benthamiana.

Supplementary Table 11 Gene list for predicted AP2 (RAV) family transcription factors in Nicotiana benthamiana.

Supplementary Table 12 Gene list for predicted AP2 (RAV) family transcription factors in Nicotiana benthamiana. 
bioRxiv preprint doi: https://doi.org/10.1101/2021.11.30.468885; this version posted December 1, 2021. The copyright holder for this preprint (which was not certified by peer review) is the author/funder, who has granted bioRxiv a license to display the preprint in perpetuity. It is made

A

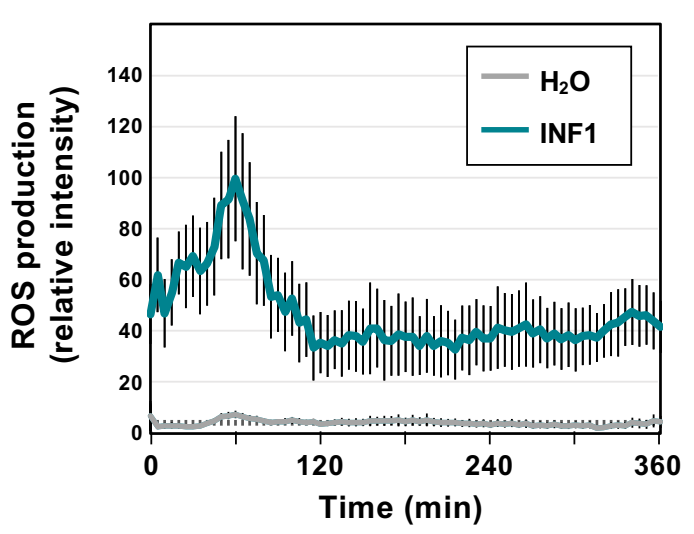

C

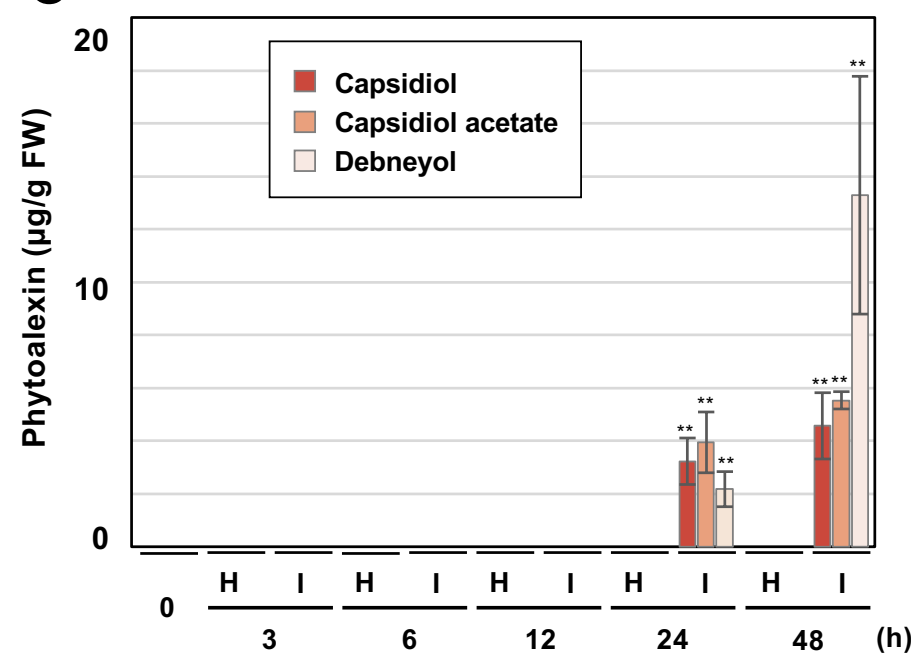

B

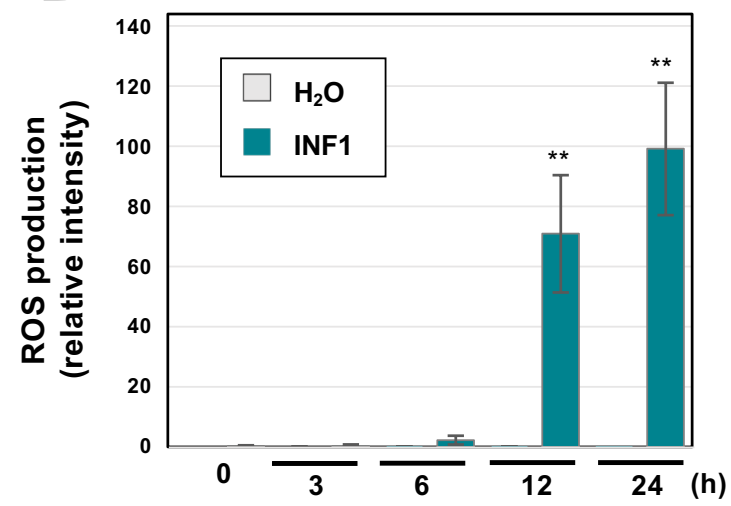

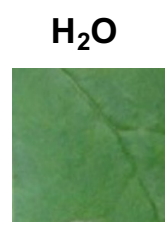

INF1

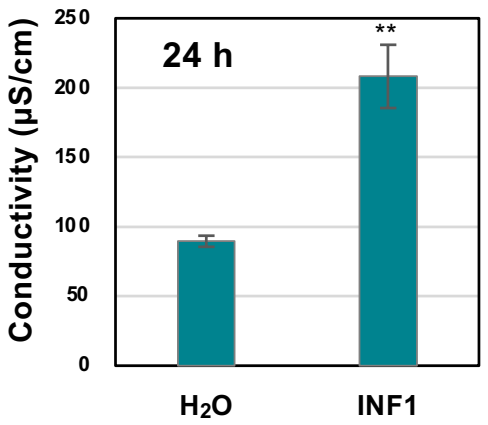

FIGURE 1 Induction of defense responses of Nicotiana benthamiana leaves treated with INF1, a secretory protein derived from Phytophthora infestans. (A) INF1-induced reactive oxygen species (ROS) production in $N$. benthamiana. Leaf discs were placed in water $\left(\mathrm{H}_{2} \mathrm{O}\right)$ or $150 \mathrm{nM}$ INF1 solution containing L-012, and L-012 mediated chemiluminescence was measured. Data are means \pm standard error (SE) $(\mathrm{n}=8)$. (B) Leaves of $N$. benthamiana were treated with water, or $150 \mathrm{nM}$ INF1 by infiltration and ROS production was detected at indicated time after the treatment. Data are means \pm standard error $(\mathrm{SE})(\mathrm{n}=6)$. (C) Phytoalexins were extracted at the indicated time after water $(\mathrm{H})$ or 150 nM INF1 (I) treatment and quantified by LC/MS. Data are means \pm SE $(\mathrm{n}=3)$. (D) Leaves of $N$. benthamiana were treated with water, or $150 \mathrm{nM}$ INF1, and electrolyte leakage was quantified at $24 \mathrm{~h}$ after INF1 treatment. Data are means \pm SE $(\mathrm{n}=6)$. Photographs were taken $72 \mathrm{~h}$ after the treatment. Data marked with asterisks are significantly different from control as assessed by the two-tailed Student's $t$-test: $* * P<0.01$. 

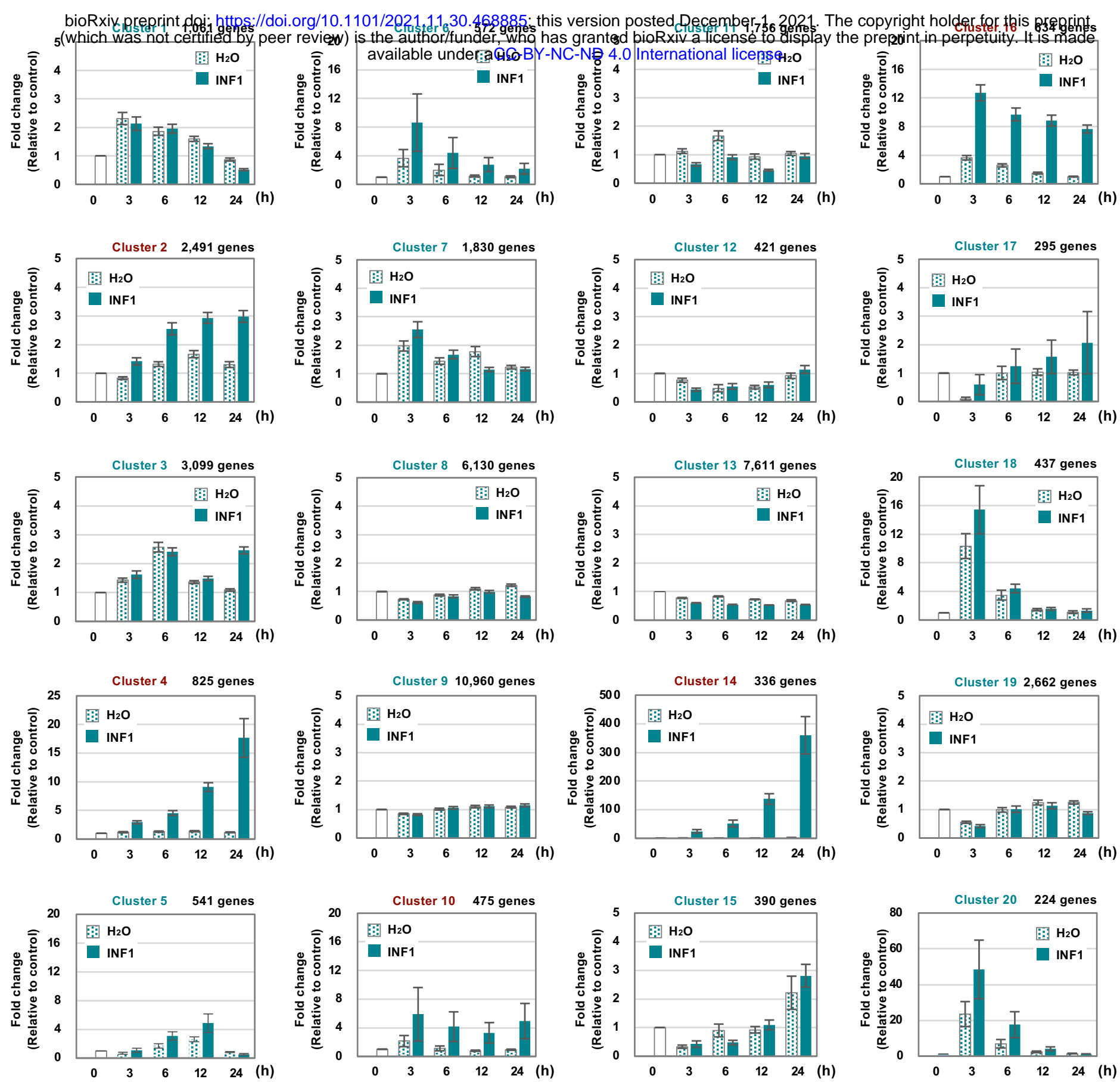

FIGURE 2 | Clustering of 57,140 Nicotiana benthamiana genes based on their expression profiles in leaves treated with water or $150 \mathrm{nM}$ INF1. $14,390(25.2 \%)$ of genes were not assigned to any cluster because no expression could be determined at any point in time. See methods for detailed procedure of the gene clustering. Graphs shown are average expression profiles of 100 genes from each cluster (the most highly expressed genes of each cluster for all treatments and timepoints). Data are means \pm SE $(n=100)$. Clusters for INF1 induced genes are shown in red letters. 
NbEAS1

NbEAS2

NbEAS3

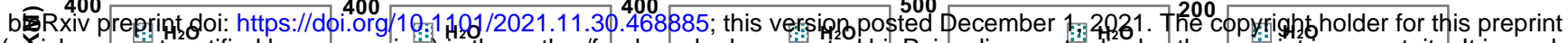
(which was not certified by peer review) is the author/funder, who has granted bioforv a license to display the preprint in perpetuity. It is made
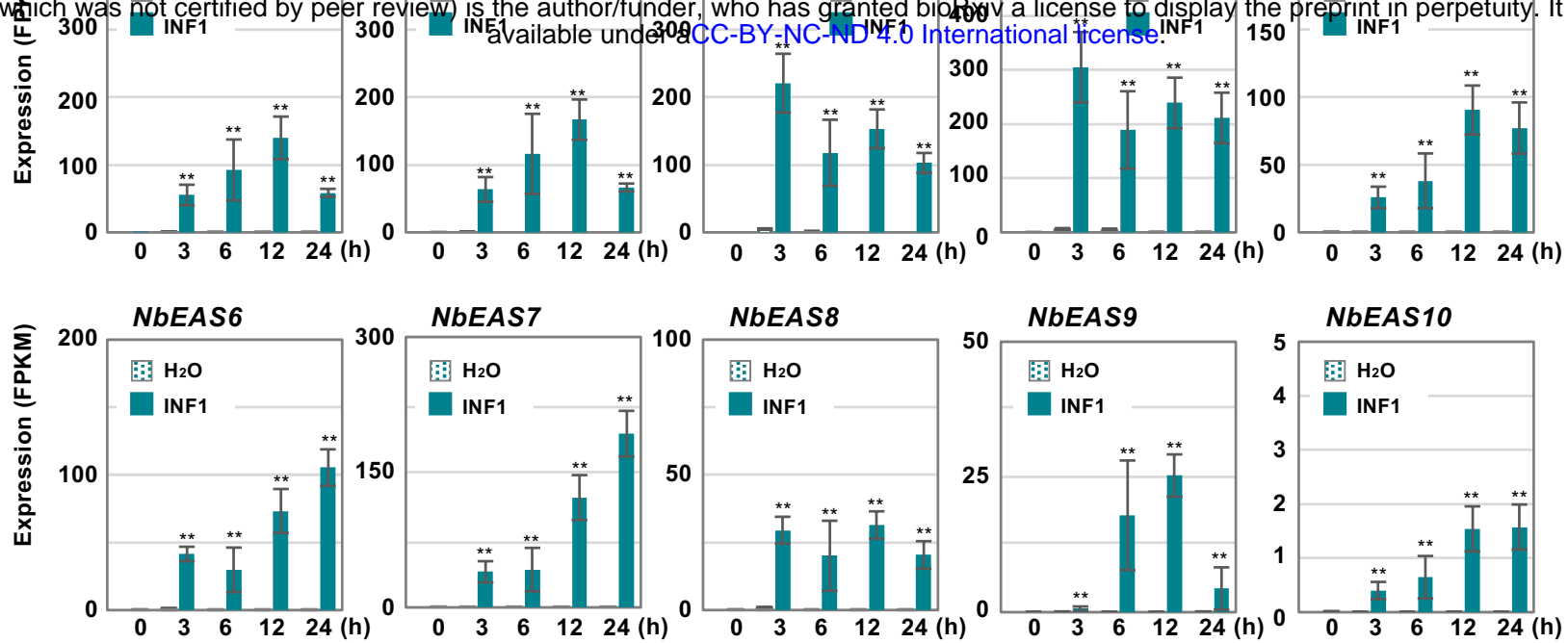

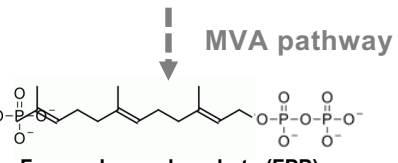

Farnesyl pyrophosphate (FPP)

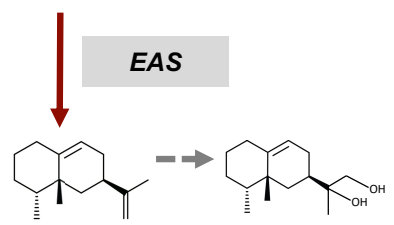

5-epi-Aristolochene (EA) Debneyol

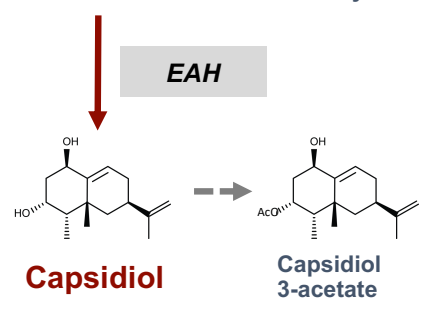

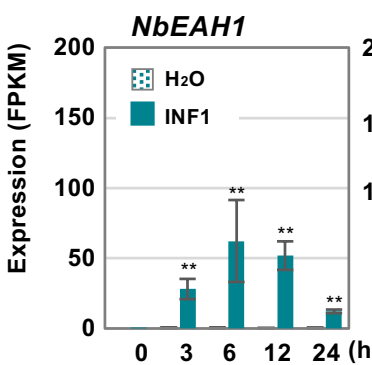
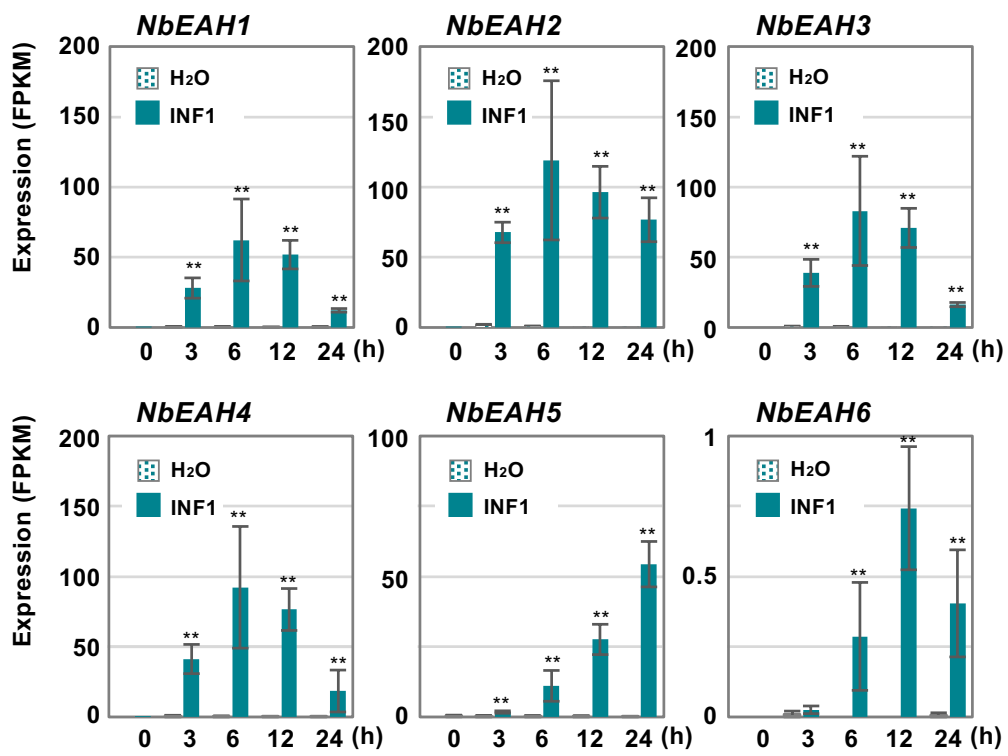

FIGURE 3 I The time course of expression of Nicotiana benthamiana genes encoding dedicated enzymes for phytoalexin production. The gene expression (FPKM value) was determined by RNA-seq analysis of $N$. benthamiana leaves treated with water or $150 \mathrm{nM}$ INF1 for $0 \mathrm{~h}, 3 \mathrm{~h}, 6 \mathrm{~h}, 12 \mathrm{~h}$ and $24 \mathrm{~h}$. Data are mean $\pm \mathrm{SE}$ $(n=3)$. Asterisks indicate a significant difference from the control (water-treated) as assessed by two-tailed Student's $t$-test, $* * P<0.01$. EAS 5-epi-aristolochene synthase, EAH 5-epi-aristolochene-1,3-dihydroxylase. 


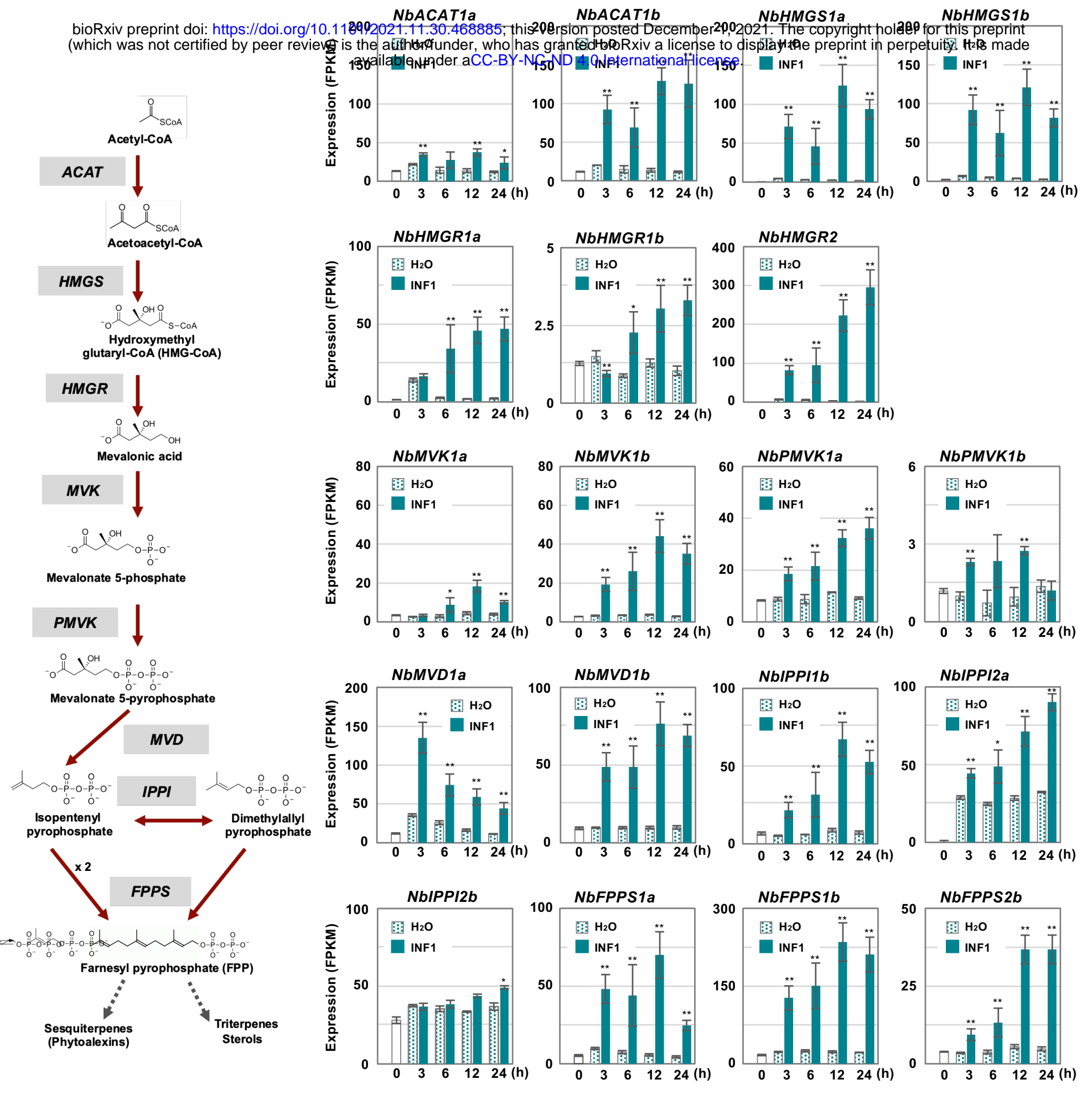

FIGURE 4| The time course of expression of Nicotiana benthamiana genes encoding enzymes in the mevalonate pathway. Gene expression (FPKM value) was determined by RNA-seq analysis of $N$. benthamiana leaves treated with water $\left(\mathrm{H}_{2} \mathrm{O}\right)$ or $150 \mathrm{nM}$ INF1 for $3 \mathrm{~h}, 6 \mathrm{~h}, 12 \mathrm{~h}$ and $24 \mathrm{~h}$. Data are means $\pm \mathrm{SE}(\mathrm{n}=3)$. Data marked with asterisks are significantly different from control as assessed by the two-tailed Student's $t$-test: $* * P<0.01 * P<0.05$.

$A C A T$, Acetoacetyl-CoA thiolase; HMGS, Hydroxymethylglutaryl-CoA synthase; HMGR, HydroxymethylglutarylCoA-reductase; $M V K$, Mevalonate-5-kinase; PMVK, Phosphomevalonate kinase; $M V D$, Mevalonate-5-pyrophosphate decarboxylase; IPPI, Isopentenyl pyrophosphate isomerase; FPPS, Farnesyl pyrophosphate synthase. 
bioRxiv preprint doi: https://doi.org/10.1101/2021.11.30.468885; this version posted December 1, 2021. The copyright holder for this preprint (which was not certified by peer review) is the author/funder, who has granted bioRxiv a license to display the preprint in perpetuity. It is made

A
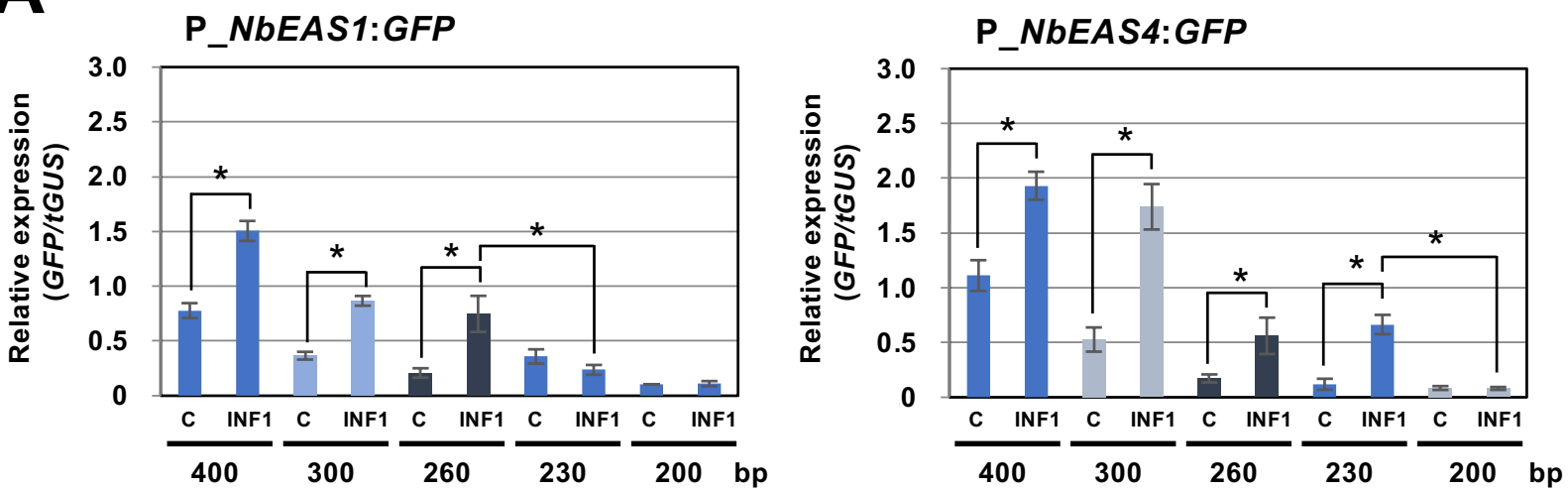

B
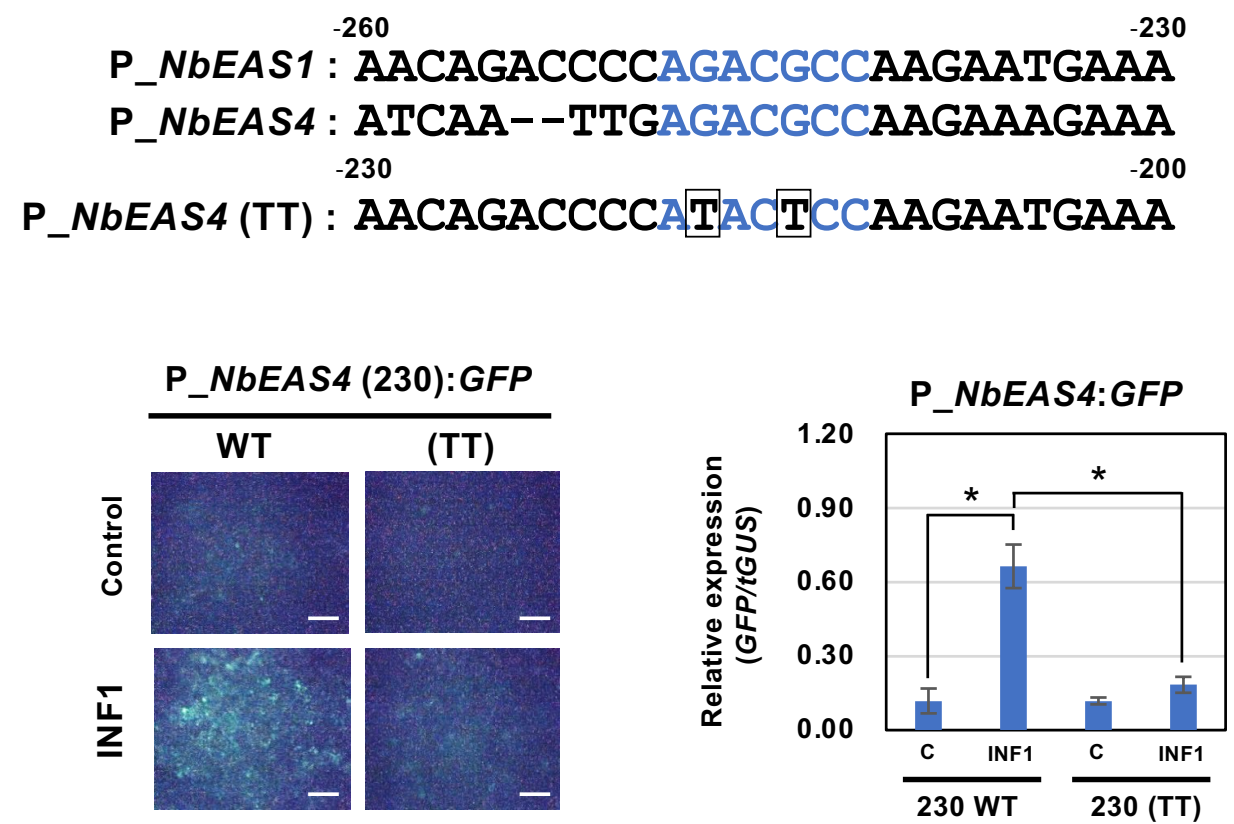

FIGURE 5 | Identification of cis-element for INF1-induced expression of $N b E A S$ genes. (A) Expression of GFP gene under the control of indicated length of NbEAS1 and NbEAS4 promoter (P_EAS). Total RNA was isolated from $N$. benthamiana leaves $48 \mathrm{~h}$ after co-inoculation with $A$. tumefaciens strains containing expression vectors for P_EAS:GFP, INF1 or control (C) vector, and truncated GUS ( $t G U S)$ gene under the control of $35 \mathrm{~S}$ promoter as internal standard. Expression of GFP gene was assessed by qRT-PCR with GFP primers and values were normalized to the expression of $t G U S$. Means $\pm \mathrm{SE}(\mathrm{n}=3)$. Data marked with asterisks are significantly different as assessed by the two-tailed Student's $t$-test: ${ }^{*} P<0.05$. (B) Alignment of -260 to -230 of NbEAS1 promoter and -230 to -200 of NbEAS4 promoter. GCC box-like motifs were shown in blue letters. Mutations introduced in NbEAS4 (TT) were shown below the alignment by boxes. (C) Expression of GFP gene under the control of $230 \mathrm{bp}$ of wild type or mutated (TT) NbEAS4 promoter. (Left) Fluorescence images of $N$. benthamiana leaves $48 \mathrm{~h}$ after $A$. tumefaciens inoculation containing indicated expression vectors. Bars $=500$ $\mu \mathrm{m}$. (Right) Total RNA was isolated from $N$. benthamiana leaves $48 \mathrm{~h}$ after co-inoculation with $A$. tumefaciens strains containing expression vectors. Expression of GFP gene was assessed by qRT-PCR with GFP primers and values were normalized to the expression of $t G U S$. Means $\pm \mathrm{SE}(\mathrm{n}=3)$. Data marked with asterisks are significantly different as assessed by the two-tailed Student's $t$-test: $* P<0.05$. 
bioRxiv preprint doi: https://doi.org/10.1101/2021.11.30.468885; this version posted December 1, 2025. ThlecGSY1/ght holder for this preprint

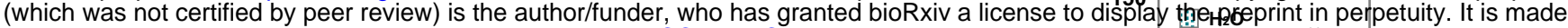

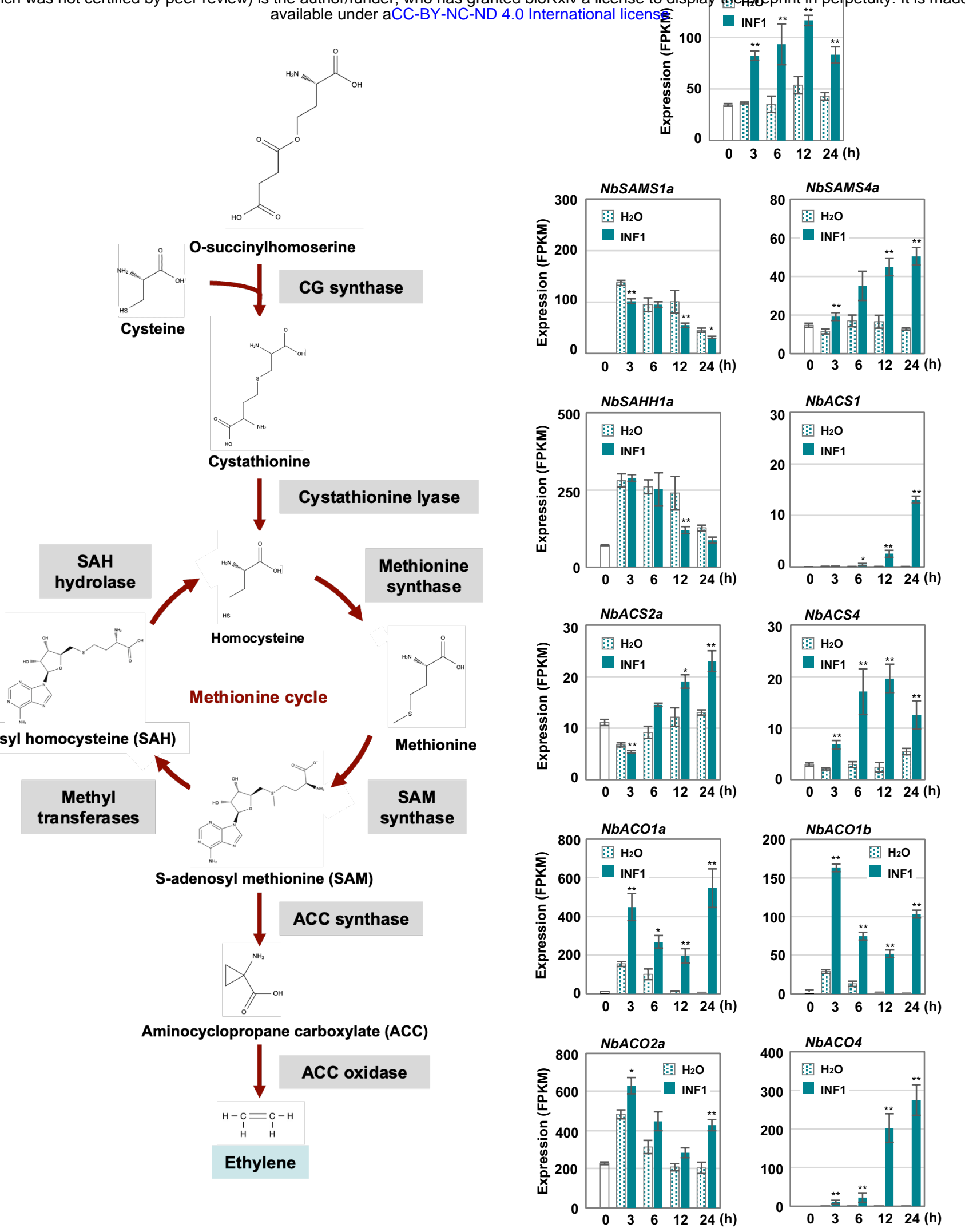

FIGURE 6 | Expression profiles of Nicotiana benthamiana genes encoding enzymes for ethylene production. The gene expression (FPKM value) was determined by RNA-seq analysis of $N$. benthamiana leaves treated with water and $150 \mathrm{nM} \mathrm{INF} 1$ for $0 \mathrm{~h}, 3 \mathrm{~h}, 6 \mathrm{~h}, 12 \mathrm{~h}, 24 \mathrm{~h}$. Data are means $\pm \mathrm{SE}(\mathrm{n}=3)$. Data marked with asterisks are significantly different from control as assessed by the two-tailed Student's $t$-test: $* * P<0.01, * P<0.05$.

CGS, Cystathionine gamma-synthase; SAMS, S-adenosylmethionine synthetase; SAHH, S-adenosylhomocycteine hydrolase; ACS, Aminocyclopropane carboxylate synthase; ACO, Aminocyclopropane carboxylate oxidase. 
bioRxiv preprint doi: https://doi.org/10.1101/2021.11.30.468885; this version posted December 1, 2021. The copyright holder for this preprint (which was not certified by peer review) is the author/funder, who has granted bioRxiv a license to display the preprint in perpetuity. It is made

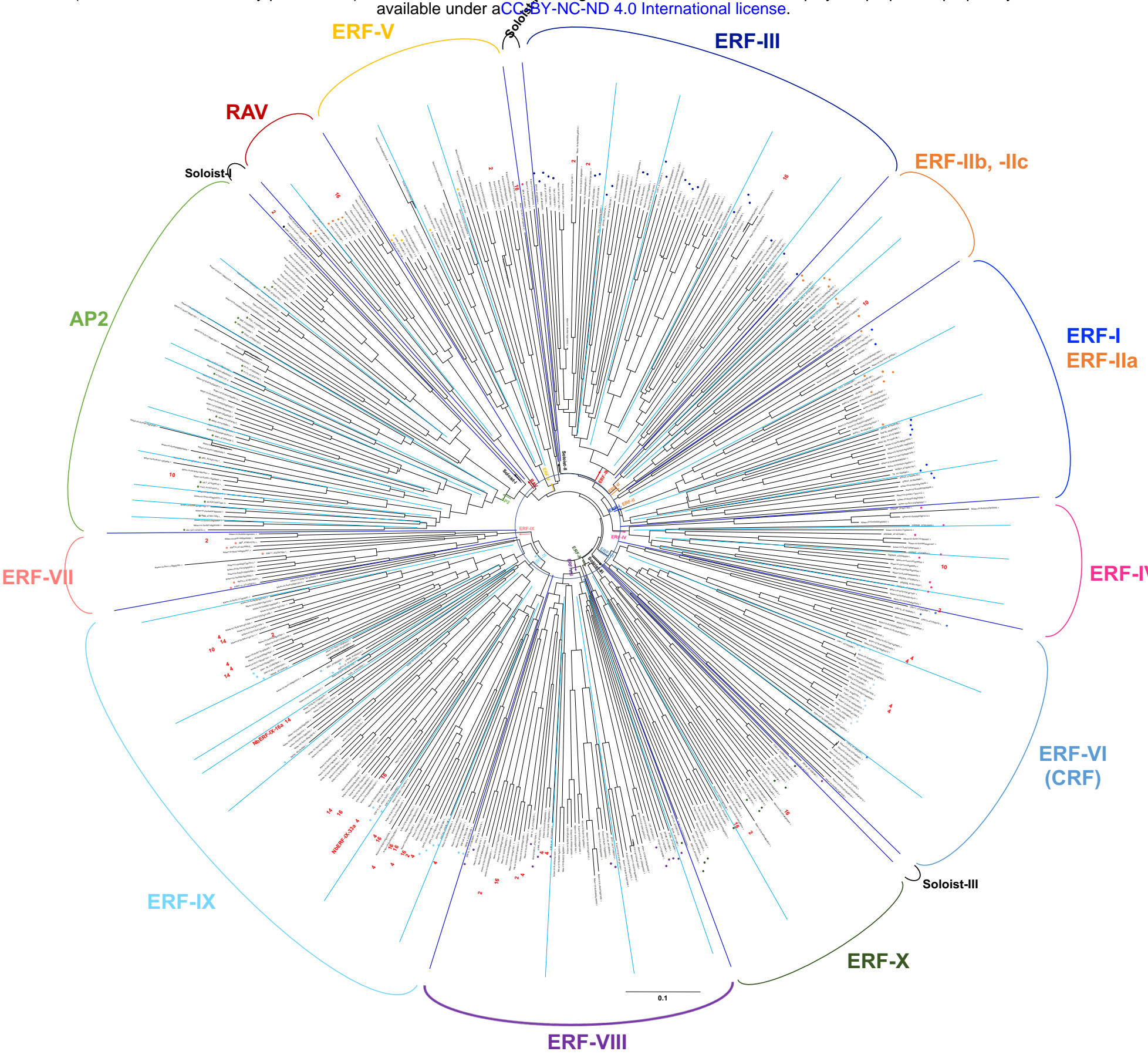

FIGURE 7 | An unrooted phylogenetic tree of AP2/ERF transcription factors form Nicotiana benthamiana and Arabidopsis thaliana. The deduced amino acid sequences of AP2/ERF transcription factors were aligned by ClustalW (Thompson et al., 1994), and the phylogenetic tree was constructed using the neighbor-joining (NJ) method (Saitou and Nei, 1987). Classification of AP2/ERF transcription factors by Nakano et al. (2006) are indicated. The cluster numbers of INF1-inducible genes are shown in red letters. The scale bar corresponds to 0.1 estimated amino acid substitutions per site. 


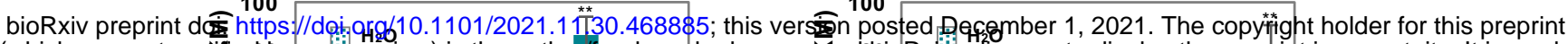

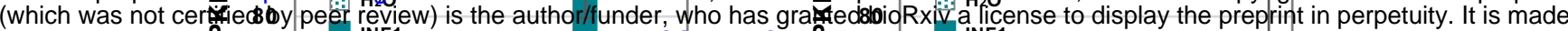
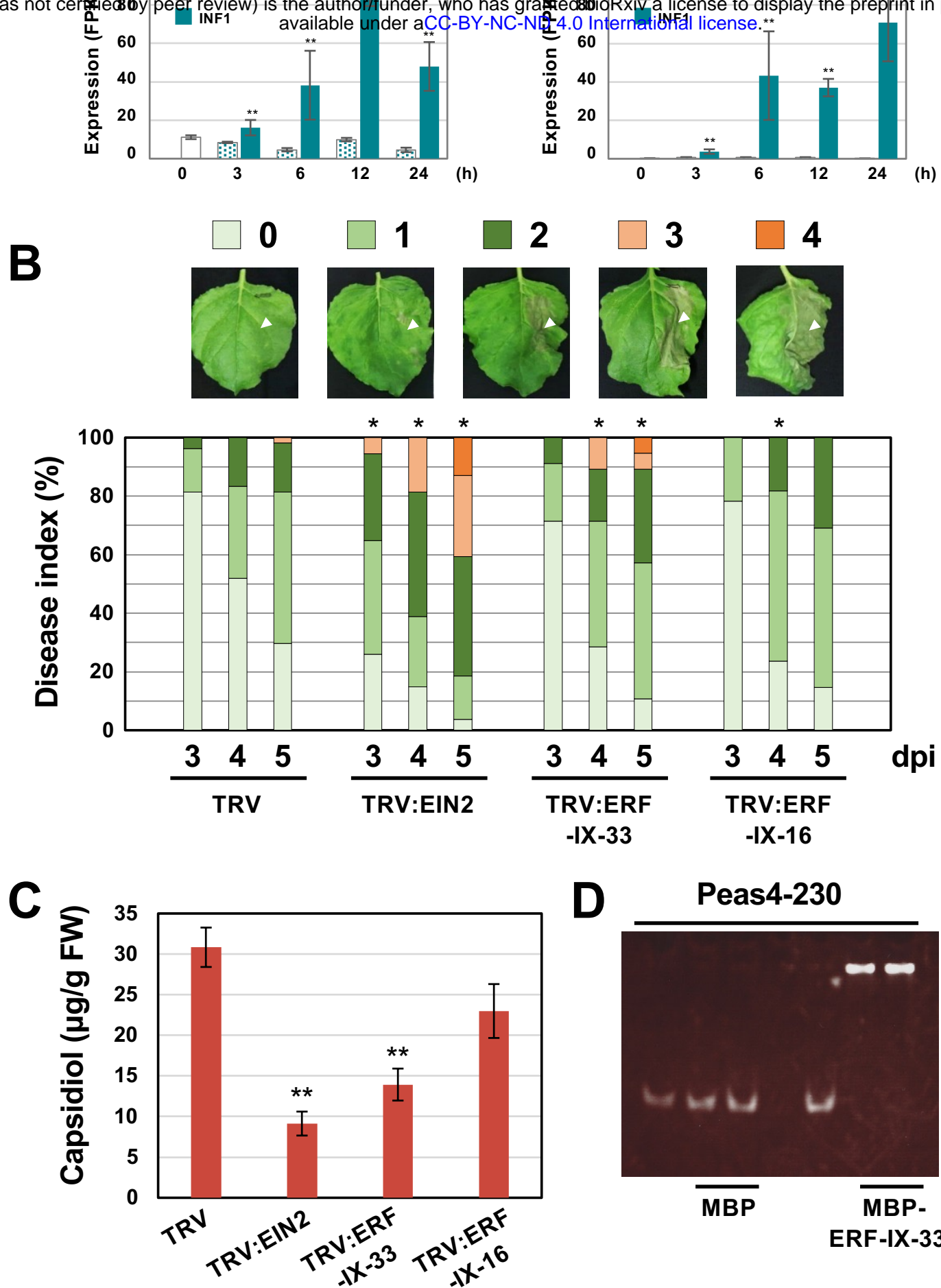

D

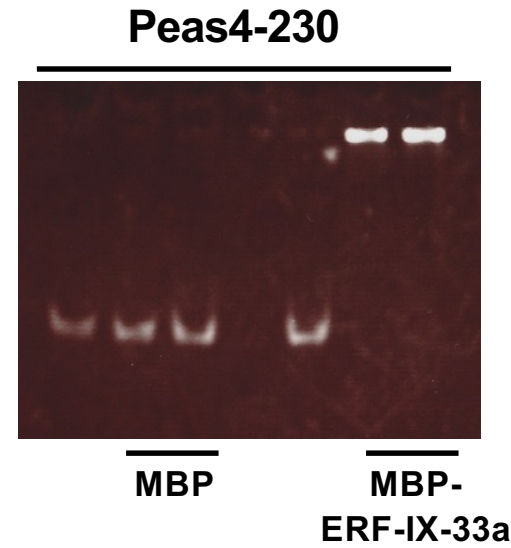

FIGURE 8 | (A) Expression profiles of Nicotiana benthamiana genes encoding two selected ERF transcription factors. Gene expression (FPKM value) was determined by RNA-seq analysis of $N$. benthamiana leaves treated with water $\left(\mathrm{H}_{2} \mathrm{O}\right)$ or 150 $\mathrm{nM}$ INF1. Data are means $\pm \mathrm{SE}(\mathrm{n}=3)$. Data marked with asterisks are significantly different from control (water-treated). $* * P<0.01$. (B) $N$. benthamiana were inoculated with TRV, TRV:ERF-IX-33 or TRV:ERF-IX-16 and the right side of leaves of control or gene-silenced plants were inoculated with $P$. infestans. The appearance of disease symptoms was categorized into 5 classes according to the severity of disease symptoms. 0 , no visible symptom; 1 , small wilted spots in inoculated area; 2 , browning $<50 \%$ of the inoculated side of the leaf; 3 , browning $>50 \%$ of inoculated side of leaf; 4 , development of disease symptoms over central leaf vein. Plot showing percentage of $N$. benthamiana leaves with disease symptom severities represented in the five classes as shown in the upper panels, for leaves of control and gene-silenced plants inoculated with $P$. infestans from 3 to 5 days post inoculation (dpi). At least 50 leaves from each control and gene-silenced plants were scored. Data marked with asterisks are significantly different from control as assessed by one-tailed Mann-Whitney $\mathrm{U}$ tests: $* P<$ 0.05. (C) Production of capsidiol in TRV-inoculated or gene-silenced $N$. benthamiana. Leaves were harvested 24 hours after $150 \mathrm{nM}$ INF1 treatment and extracted phytoalexins were detected by HPLC. Data are means \pm SE $(\mathrm{n}=4)$. Data marked with asterisks are significantly different from control. ${ }^{*} P<0.01$. (D) Binding of NbERF-IX33a to the NbEAS4 promoter. MBP (maltose binding protein) or MBP-ERF-IX-33a was incubated with NbEAS4 promoter fragments. Samples were separated in a non-denaturing polyacrylamide gel. The gel was stained with SYBR Green EMSA stain. 